\title{
Klaus Busch \\ Die Europäische Gemeinschaft in der Krise \\ - die Ursachen des Scheiterns der Wirtschafts- und Währungsunion
}

\section{Der bisherige Verlauf der Integration in der Europäischen Gemeinschaft: Von der Zollunion zur Wirtschafts- und Währungsunion}

Der westeuropäische Integrationsprozeß muß im Kontext des innerimperialistischen Konkurrenzkampfes zwischen den USA und Westeuropa analysiert werden. Da der Produktivitätsvorsprung des USA-Kapitals gegenüber seinen europäischen Konkurrenten auf die amerikanischen Binnenmarktsverhältnisse zurückzuführen ist, die dem amerikanischen Kapital einen vergleichsweise hohen Konzentrations- und Zentralisationsgrad und damit verbunden ein vergleichsweise hohes technologisches Niveau ermöglichen, versucht das westeuropäische Kapital diese Konkurrenznachteile durch einen sukzessiven Integrationsprozeß seiner nationalen Märkte aufzuheben. Erklärtes Ziel der EG ist nach den Römischen Verträgen von 1958 die Bildung einer Wirtschaftsgemeinschaft mit freiem Waren- und Kapitalverkehr, der Freizügigkeit der Arbeitskräfte, der Niederlassungsfreiheit nach innen und einer gemeinsamen Handelspolitik nach außen.

Diese Strategie war in den 60er Jahren, als es vor allem um die Verwirklichung der Zollunion, den Gemeinsamen Markt, ging, sehr erfolgreich. Die Zollmauern zwischen den 6 Mitgliedsländern wurden bis zum Juli 1968 schrittweise abgebaut, so daß sich der Konkurrenzkampf zwischen den nationalen europäischen Kapitalen intensivierte. Während sich das Sozialprodukt (in laufenden Preisen) von 1958 bis 1968 etwas mehr als verdoppelte, vervierfachte sich im selben Zeitraum (in laufenden Preisen) der Handel innerhalb der EWG. Gleichzeitig wuchs der Handel der EWG mit der übrigen Welt um $110 \%$, d.h. im selben Tempo wie der Welthandel insgesamt (1), Der Anteil des EWG-Intrahandels am EWG-Gesamthandel, der sogenannte Integrationskoeffizient, stieg von $195830,1 \%$ auf $196845,0 \%$ und erreichte 1972 bereits $50,1 \%(2)$.

Infolge des verschärften Konkurrenzkampfes der nationalen Kapitale in einem gemeinsamen europäischen Markt ist der Konzen trations- und Zentralisationsprozeß des Kapitals verschärft worden (wenn auch zunächst hauptsächlich im Rahmen der einzelnen Nationen), so daß die Zollunion insgesamt dem Akkumulationsprozeß des westeuropäischen Kapitals erhebliche Impulse verlieh. Nicht zuletzt auf diesen F aktor ist es auch zurückzuführen, daß die Produktivitätslücke zwischen dem USA-Kapi-

1 Pierre Maillet u.a.: Die Europäische Wirtschaftsgemeinschaft im Ausbau, Baden-Baden 1972, S. 54

2 Günter Heiduk: Integrationseffekte - Theorie und Praxis, in: Wirtschaftsdienst Nr. 6, 1975, S. 305. 
tal und den westeuropäischen Kapitalen in den 60er Jahren enger wurde. Das USABruttoinlandsprodukt (zu Marktpreisen) je Erwerbstätigen wuchs von 1960 bis 1969 um $23 \%$, gleichzeitig stieg die so gemessene Produktivität pro Erwerbstätigen in der EG um $54 \%$. Der absolute Produktivitätsvorsprung der USA vor der EG war damit vom Verhältnis 3:1 auf die Relation 2,5:1 gesunken.

In den 70er Jahren soll diese Strategie des westeuropäischen Kapitals darin ihre Fortsetzung erfahren, daß mit Großbritannien, Irland und Dänemark die gemeinschaftliche Zollunion (Endetappe 1977) um 3 Länder erweitert wird und mit den restlichen EFTA-Ländern - Österreich, Schweiz, Schweden, Finnland, Island und Portugal - eine westeuroäische Freihandelszone gegründet wird (Endetappe ebenfalls 1977).

Während sich die Zollunion in den 60er Jahren also relativ reibungslos verwirklichen ließ, scheiterte die anvisierte nächste Stufe des Integrationsprozesses, die Bildung einer Wirtschafts- und Währungsunion (Beschluß der Haager Gipfelkonferenz vom Dezember 1969), von Anbeginn.

Ziel der Wirtschafts- und Währungsunion (WWU) ist die vollständige Liberalisierung des Waren-, Arbeitskräfte- und Kapitalverkehr in einem EG-Währungssystem, das durch die irreversible Konvertibilität der Währungen, die unwiderrufliche Fixierung der Wechselkursparitäten, die Beseitigung der Wechselkursbandbreiten, sowie ein einheitliches und gemeinschaftliches Zentralbanksystem chrakterisiert ist. Nach langen Auseinandersetzungen über die sinnvollste Konzeption zur schrittweisen Verwirklichung der WWU beschloß der EG-Ministerrat im Frühjahr 1972 folgenden Stufenplan:

In einer ersten Stufe (rückwirkend vom 1.1.1971 bis zum 31.12.1973) sollen die Konsultationen der Mitgliedsländer über die jeweilige Wirtschaftspolitik intensiviert und als erster währungspolitischer Schritt die Bandbreiten zwischen den Wechselkursen der einzelnen Länder auf 2,25\% begrenzt werden. Gleichzeitig ist im Verlauf der ersten Etappe das Währungsbeistandssystem in der EG auszubauen, mit dessen Hilfe die Wechselkursparitäten im beschlossenen Rahmen fixiert werden sollen. Nach Ablauf dieser ersten Stufe zur WWU, die gegebenenfalls um 2 Jahre verlängert werden kann, hat der Ministerrat Maßnahmen für den Übergang zur zweiten Etappe $\mathrm{zu}$ beschließen. Kernstück der zweiten Stufe soll die Ausweitung des währungspolitischen Beistandssystems sowie die Abstimmung der mittelfristigen Wirtschaftspolitik der Mitgliedsländer sein. Die weiteren Stufen der WWU sind durch Beschlüsse des Ministerrates festzulegen, wobei mit einer immer enger werdenden Koordinierung der Wirtschaftspolitik schließlich die Bedingungen für eine endgültige Wechselkursunion geschaffen werden sollen. Als Gesamtzeitraum für die Installierung der WWU sind 10 Jahre vorgesehen.

Es ist verständlich, daß das WWU-Konzept auf ein weitaus größeres Maß an nationalen Widerständen stößt als die Bildung der Zollunion. Während die Zollunion durch den wechselseitigen Abbau von Importbarrieren jedem EG-Land Vor- und Nachteile brachte und im Falle zu einseitiger Benachteiligung eines Landes, die sich sehr rasch in Handelsbilanzdefiziten bemerkbar gemacht hätte, die Möglichkeit der Wechselkurskorrektur offen ließ, bevorzugt die WWU die nationalen Kapitale mit 
stärkeren Produktivitätsfortschritten einseitig und beraubt die sich schwächer entwickelnden nationalen Kapitale der Möglichkeit, Veränderungen der Konkurrenzbedingungen im EG-Raum mit Wechselkurskorrekturen aufzufangen. Das Ausmaß der ungleichen Entwicklung der Konkurrenzbedingungen im EG-Raum indiziert die ungleiche Entwicklung der Durchschnittswerte der Ausfuhr für die wichtigsten EG-Länder. Während dieser Index für die Preisentwicklung der Exportgüter von 1963 gleich 100 bis 1970 für die BRD auf 103 anstieg, erreichte er für Italien 107, für Frankreich auf 147, für Großbritannien auf 159 und für Italien auf 180 (3). Fixierte Wechselkurse im Rahmen einer WWU hätten unter diesen Bedingungen für das britische und das italienische Kapital verheerende Wirkungen.

Kein Wunder, daß bereits die erste Stufe der Wirtschafts- und Währungsunion faktisch scheiterte. Nachem im März 1972 alle EG-Länder die Bandbreite ihrer Währungen untereinander von $4,5 \%$ auf $2,25 \%$ verringerte und sich die beitrittswilligen europäischen Länder (Großbritannien, Irland, Dänemark und Norwegen) diesem Schritt anschlossen, scherten 1 Jahr später mit dem Übergang der EG-Währungen zum Blockfloaten gegenüber dem Dollar Großbritannien, Irland und Italien aus dem Währungsverbund aus. Die DM wurde 1973 innerhalb des Blocks um zunächst $3 \%$ und dann abermals um 5,5\% aufgewertet. Frankreich scherte von Januar 1974 bis zum Juli 1975 aus dem Euroblock aus, so daß in diesem Zeitraum von den 9 EG-Ländern nur noch 5 (die BRD, die Beneluxländer und Dänemark) ihre Währungen aneinander gebunden hatten. Auch die Rückkehr des Franc in den europäischen Währungsblock im Juli 1975 änderte nichts am Scheitern der Wechselkursunionpläne, denn Italien, Irland und Großbritannien ließen ihre Währungen weiterhin unabhängig von den europäischen Partnerwährungen floaten und eine ungleichmäßige Akkumulationsentwicklung in den restlichen sechs Mitgliedsländern konnte diesen Währungsverbund jederzeit sprengen. Inzwischen ist der Franc im März 1976 erneut aus dem europäischen Währungsverbund ausgeschieden.

Die Fragen, die sich angesichts dieses Scheiterns bereits der ersten Etappe zur Wirtschafts- und Währungsunion aufdrängen, lauten:

1. Sind die Realisierungsschwierigkeiten der WWU nur die üblichen und historisch bekannten Geburtswehen des europäischen Integrationsprozesses, die am Ende doch überwunden werden können:

„Es ist einfach, das Ende der nationalen Währungs- und Stabilitätspolitik vorauszusagen. Aber die Erkenntnis, daß3 die nationalen Alleingänge der europäischen Staaten letztlich unwirksam bleiben, setzt sich nur langsam durch. Vorerst erfolgt der Lern- und Anpassungsprozeß der Regierungen und Bürokratien, der Sozialpartner, Unternehmer und Verbraucher eher über schmerzhafte Krisen, denn über die Einsicht in die gegenseitige Abhängigkeit und in die Notwendigkeit einer wirtschaftlichen und politischen Integration. Da die Flucht in die nationale Abkapselung kein gangbarer Ausweg mehr ist, bleibt nur, den kühnen Plan der WWU zu verwirklichen, damit Europa als selbständige und vielfältige Einheit überleben kann." (4)

2. Ist die Krise der WWU nur die Krise des verfrühten monetären Integrations-

$3 \quad$ Jahresgutachten des Sachverständigenrates zur Begutachtung der gesamtwirtschaftlichen Entwicklung 1970. 1972, 1975, S. 176 f, S. 202 f, S. 223

4 Wegener, Manfred, Wirtschafts- und Währungsunion - Ziele und Wege, in: Weinstock, Ulrich (Hrsg.): Neun für Europa, Düsseldorf 1973, S. 90 f. 
konzeptes, das Wechselkursstabilität verlangte, ehe die Wirtschaftspolitik auch nur ansatzweise koordiniert werden konnte?

3. Läßt sich durch eine Abstimmung der Wirtschaftspolitik der EG-Mitgliedsstaaten der ökonomische Verlauf in der EG harmonisieren? Oder würden auch bei einer supranationalen Wirtschaftspolitik und einem europäischen Zentralbanksystem strukturelle Faktoren eine ungleichmäßige Akkumulationsentwicklung in der EG produzieren, die das WWU-Konzept von daher grundsätzlich als fragwürdig erscheinen lassen?

Diese Fragen können erst nach einer gründlichen Untersuchung der Bedingungen des Akkumulationsverlaufes in den wichtigsten EG-Staaten beantwortet werden, die im weiteren Verlauf dieses Aufsatzes im Mittelpunkt steht. Erst eine Analyse der Ursachen des ungleichmäßigen Akkumulationstempos in der EG kann die Frage nach dem zufälligen oder notwendigen Scheitern der WWU fundiert beantworten. Besonderes Gewicht ist im Rahmen der Akkumulationsuntersuchung auf die Ursachen der ungleichen Inflationsraten in der EG zu legen, dem Inflationsproblem wird deshalb ein gesonderter Abschnitt gewidmet. Angesichts des zu bewältigenden quantitativen Materials beschränkt sich die Analyse der ungleichen Entwicklung in der EG auf die vier wichtigsten Mitgliedsländer Großbritannien, Italien, Frankreich und die BRD (5).

\section{Die ungleichmäßige Kapitalakkumulation in der EG}

\subsection{Theoretische und methodische Vorüberlegungen zur Untersuchung der ungleichmäßigen Entwicklung}

Bevor im Verlaufe dieses Kapitels der reale ungleiche Akkumulationsprozeß in den vier EG-Ländern, Italien, Großbritannien, Frankreich und BRD, analysiert wird, sollen einleitend einige theoretische Prämissen über die Ursachen ungleichmäßiger Kapitalakkumulation sowie die Gründe für die Auswahl der in diesem Aufsatz angewandten Indikatoren der Kapitalverwertungsbedingungen dargestellt werden.

Zunächst zur Theorie der ungleichmäßigen Kapitalakkumulation: Unter kapitalistischen Produktionsbedingungen sind die Profitabilität der Produktion und die Konkurrenz die Haupthebel der Entfaltung der Akkumulation. Günstige Kapitalverwertungsbedingungen sind die erste Voraussetzung eines kontinuierlichen Akkumulationsprozesses. Für die Analyse des Akkumulationstempos ist diese Bestimmung aber nicht ausreichend; hinzu kommt das Ausmaß des Konkurrenzkampfes, dem das Kapital ausgesetzt ist. Kapitale, die über die gleichen günstigen Verwertungsbedingungen verfügen, werden ungleich akkumulieren, wenn der Zwang zur Akkumulation aufgrund eines unterschiedlichen Konkurrenzdruckes, dem sie ausgeliefert sind, 
ein divergierender ist (6). Erst die Verbindung von Verwertungs- und Konkurrenzbedingungen läßt in der Akkumulationsanalyse Schlüsse auf das Entwicklungstempo der Kapitalakkumulation zu. In der Untersuchung der Ursachen der ungleichmäßigen Kapitalakkumulation zwischen verschiedenen nationalen Kapitalen ist also zunächst nach den Ursachen ungleicher Verwertungsbedingungen Ausschau zu halten, um dann in einem zweiten Schritt zu analysieren, ob sich in unterschiedlichen Konkurrenzbedingungen weitere Faktoren für die ungleichmäßige Entwicklung erschlieBen lassen.

Betrachten wir zunächst die Kapitalverwertungsbedingungen, so ist aufgrund der allgemeinen Gesetze der Kapitalakkumulation davon auszugehen, daß die entwickeltsten nationalen Kapitale über die geringsten nationalen Durchschnittsprofitraten verfügen, und umgekehrt die Länder mit geringerem absoluten Akkumulationsniveau die höchsten durchschnittlichen nationalen Profitraten realisieren. Aufgrund der niedrigeren nationalen Verwertungsraten wird das Akkumulationstempo der höher entwickelten kapitalistischen Länder geringer sein als das der schwächer entwikkelten nationalen Kapitale.

Die Prämisse von der negativen Korrelation zwischen absolutem Akkumulationsniveau und Höhe der Durchschnittsprofitrate ergibt sich aufgrund folgender drei Überlegungen:

1. In einem Land mit höherem Akkumulationsniveau wird in der Regel auch die organische Zusammensetzung des Kapitals im internationalen Vergleich überdurchschnittlich hoch liegen. Die höhere Kapitalzusammensetzung dieses Landes drückt dessen nationale Durchschnittsprofitrate und damit dessen Zuwachsraten in der Kapitalakkumulation nach unten. Einen anderen Ausdrucken finden die unterschiedlichen Akkumulationsbedingungen ungleich entwickelter Länder darin, daß in der höher entwickelten Nation der Kapitalkoeffizient $\left(\frac{\mathrm{K}}{\mathrm{Y}}\right)$ einen höheren Wert erreicht als in der schwächer entwickelten Nation und umgekehrt die Kapitalproduktivität $\left(\frac{\mathrm{Y}}{\mathrm{K}}\right)$ in der entwickelteren Nation niedriger ist. Ein wachsender Kapitalvorschuß pro produzierter Werteinheit $\left(\frac{\mathrm{K}}{\mathrm{Y}}\right)$ produziert bei konstantem Teilungsverhältnis des Wertproduktes in gesellschaftlich notwendige und Mehrarbeit eine niedrigere 
durchschnittliche Verwertungsrate und so bescheidenere Akkumulationszuwachsraten.

2. Ein weiterer wichtiger Faktor für die ungleichmäßige Akkumulation ist in der ungleichen sektoralen Struktur ungleich entwickelter Ökonomien zu sehen. In den schwächer entwickelten Ländern hat der Industriesektor im Vergleich zur Landwirtschaft und zum Dienstleistungsbereich noch nicht einen so hohen Anteil an der Entstehung des Wertproduktes erreicht wie in den höher entwickelten Ländern. Die schrittweise Auflösung vorkapitalistischer Sektoren in Landwirtschaft, Handwerk und Handel stellt insofern eine beträchtliche Akkumulationsreserve der schwächer entwickelten Länder dar, als durch die zunehmende Zerstörung weniger produktiver Bereiche im Landwirtschafts- und Dienstleistungssektor eine nationale Reservearmee an Arbeitskräften produziert wird. Diese Reservearmee begïnstigt die nationalen. Verwertungsbedingungen doppelt. Sie läßt einersetis die Mehrwertrate durch einen Druck auf den Preis der Ware Arbeitskraft anwachsen und sie sorgt andererseits für eine disponible Arbeitermasse im erweiterten Reproduktionsprozeß. Die industrielle Kapitalakkumulation kann damit leichter auf gleicher technologischer Stufenleiter stattfinden als in Ländern ohne größere Reservearmee, wo der höhere Preis der Ware Arbeitskraft und der Arbeitskraftmangel zur forcierten Substitution von Arbeitskraft durch Maschinerie mit Hilfe neuerer Technologie zwingt und damit in der Regel zu einem schnelleren Wachstum der organischen Zusammensetzung des Kapitals führt.

3. Auch die Struktur der Verteilungsverhältnisse begünstigt im Durchschnitt die Kapitalakkumulation in den schwächer entwickelten Ländern im Vergleich zu den höher entwickelten Nationen. Obwohl davon auszugehen ist, daß aufgrund des höheren Produktivitätsniveaus der notwendige Teil des Arbeitstages in den höher entwickelten Ländern geringer ist und von daher die Mehrwertrate dort höher steht, verursachen mit wachsendem Akkumulationsniveau drei Momente die schrittweise Verschiebung der Profit-Lohn-Relation zuungunsten der Profite. Zum ersten ist der Druck der Reservearmee auf die Kampfkraft der Arbeiterklasse in den entwickelteren Ländern mit höherem Beschäftigungsniveau zumeist geringer als in den weniger produktiven Nationen, in denen proletarisierte Bauern, Handwerker und Händler den Arbeitsmarkt relativ stärker speisen als neue Arbeitsplätze im erweiterten industriellen Reproduktionsprozeß des Kapitals entstehen. Zum zweiten wächst mit zunehmender Durchkapitalisierung eines Landes der relative Anteil der Lohnabhängigen an den Erwerbstätigen, so daß die tatsächliche Lohnquote im höher entwickelten Land größer ist als im schwächer entwickelten Land. Selbst wenn nun die bereinigte Lohnquote im höher entwickelten Land stagnieren sollte, tangiert dennoch das Anwachsen der tatsächlichen Lohnquote (durch die Steigerung des Lohnarbeiteranteils an den Erwerbstätigen) die Durchschnittsprofitrate negativ, weil ein wachsender Teil des Wertproduktes von den Lohnabhängigen auf Kosten der Profitquote angeeignet wird. Zum dritten nimmt mit der Durchkapitalisierung eines Landes der relative Anteil der unproduktiven Arbeiter am Gesamtarbeiter zu, wodurch ein stets wachsender Teil des Mehrwertfonds als Lohn für unproduktive Arbeit verausgabt und die Profit-Lohn-Relation zugunsten des Lohnanteils verschoben wird. 
Modifikationen von diesen objektiv bestimmten Profit-Lohn-Relationen in ungleich entwickelten Ländern ergeben sich durch das unterschiedliche Kampfbewußtsein der Arbeiterklasse in den verschiedenen Ländern. Die objektive Bestimmung der Verteilungsrelation ermöglicht von daher nur eine grobe Einschätzung der Profit-Lohn-Relationen, deren konkrete Ausprägungen in bestimmten historischen Situationen durch den divergierenden subjektiven Faktor stark vom objektiv zu erwartenden Verhältnis abweichen kann.

Aufgrund des unterschiedlichen Niveaus in der organischen Zusammensetzung des Kapitals, der ungleichen sektoralen Struktur der Ökonomie und des ungleichen Teilungsquotienten des Wertproduktes können also unterschiedliche Kapitalverwertungsbedingungen zwischen Nationen mit ungleichem absolutem Akkumulationsniveau erwartet werden. Diese ungleichen Verwertungsbedingungen werden sich in ungleichen Veränderungsraten der Kapitalakkumulation niederschlagen und aufgrund höherer Wachstumsraten in den schwächer entwickelten Ländern Angleichungsprozesse im vorhandenen Akkumulationsgefälle produzieren. In welchem Maße allerdings die ungleichen Durchschnittsprofitraten auf die Wachstumsraten des Wertproduktes durchschlagen, hängt davon ab, welchem Konkurrenzdruck die verschieden entwickelten nationalen Kapitale ausgesetzt sind. So ist davon auszugehen, daß ein schwächer entwickeltes Land seine potentiell günstigeren Akkumulationsbedingungen gegenübet einem höher entwickelten Land nicht im höchstmöglichen Maße in Wachstumsratendifferenzen ummünzen kann, wenn der Konkurrenz'druck, der auf das nationale Kapital dieses Landes ausgeübt wird, schwach entwickelt ist. Als Maßstab für den Grad des Konkurrenzkampfes in den verschiedenen Ländern kann uns dabei der Grad der Weltmarktintegration der einzelnen nationalen Kapitale dienen. Je stärker sich ein nationales Kapital durch protektionistische Maßnahmen vom Weltmarkt abgeschirmt hat, desto geringer wird der Konkurrenzdruck sein, dem die Kapitale dieses Landes ausgesetzt sind. Umgekehrt wird in den Ländern, die ihr Kapital durch keinerlei Zölle, Importkontingentierungen oder nichttarifäre Handelshemmnisse vom Weltmarkt abschotten, aufgrund des hohen Konkurrenzdruckes der Zwang zur forcierten Entfaltung der Produktivkräfte am stärksten durchschlagen. Weichen die Kapitalverwertungsbedingungen zweier nationaler Kapitale nur geringfugig voneinander ab, kann der unterschiedliche Konkurrenzdruck, der in beiden Ländern aufgrund eines abweichenden Grades der Weltmarktintegration vorherrscht, durchaus zu sehr unterschiedlichen Wachstumsraten des Wertproduktes beider Nationen führen. Die Investitionsquote des Landes mit höherer Weltmarktintegration wird unter diesen Bedingungen größer sein als die des protektionistischeren Landes.

Bevor nun auf der Basis dieser Hypothesen der empirische Akkumulationsverlauf in den EG-Ländern Italien, Großbritannien, Frankreich und BRD analysiert werden wird, ist zunächst das Indikatorensystem darzustellen, mit dessen Hilfe in dieser Arbeit die Verwertungsbedingungen des Kapitals gemessen werden.

Als Indikator für die Entwicklung der Verwertungsbedingungen in den EG- 
Ländern wird in dieser Untersuchung die marginale Kapitalrentabilität benutzt. $\Delta \mathrm{r}$ ist das Produkt aus marginaler Kapitalproduktivität $\left(\frac{\Delta Y}{\text { I }}\right)$ und marginaler Gewinnquote $\left(1-\frac{\Delta L}{\Delta Y}\right): \Delta r=\frac{\Delta Y}{I} \cdot\left(1-\frac{\Delta L}{\Delta Y}\right)$. Die Verwendung der durchschnittlichen Kapitalrentabilität $r=\frac{Y}{K} \cdot\left(1-\frac{\mathrm{L}}{\mathrm{Y}}\right)$ anstelle der marginalen Kapitalrentabilität scheitert in der EG-Analyse an unzureichenden statistischen Materialien über das Anlagevermögen (Kapitalstock) in den verschiedenen Ländern. Erstens: die Zeitreihen der Schätzungen des Anlagevermögens enden zu unterschiedlichen Zeitpunkten am Ende der 60er Jahre. Zweitens: die Preisbasis für diese Schätzungen des Kapitalstockes in den verschiedenen Ländern ist nicht einheitlich. Drittens: das wichtigste Hindernis für einen internationalen Kapitalproduktivitätsvergleich, die Schätzungen des Kapitalstockes beruhen von Land zu Land auf unterschiedlichen Methoden. Hier ist einmal das Bewertungsproblem relevant. Je nach Bewertungsansatz (zum Anschaffungswert, zum Marktwert, zum Wiederbeschaffungswert usw. ) divergieren die absoluten Kapitalstockwerte erheblich und führen zu unterschiedlichen Kapitalproduktivitätsdaten (7).

Zum anderen basieren die Schätzungen des Anlagevermögens auf unterschiedlichen Überlebensfunktionen des Kapitalstockes, also unterschiedlichen Abschreibungsmethoden, so daß auch von dieser Seite her die absoluten Werte des Anlagevermögens stark voneinander abweichen können.

Wie prekär das Datenproblem bei internationalen Kapitalstockvergleichen ist, faßt Schatz wie folgt zusammen:

„.. Kapitalstockberechnungen liegen nur für eine begrenzte Anzahl von Ländern vor. Einer OECD-Studie kann man entnehmen, daß für nur 12 der OECD-Länder Bruttokapitalstockdaten überhaupt verfügbar sind, darunter vielfach private Schätzungen. Die Schätzmethoden differieren; nur 5 der Zeitreihen reichen über das Jahr 1960 hinaus. Längere, bis in die Gegenwart reichende Zeitreihen sind noch knapper; zudem beruhen die Schätzungen für den realen Kapitalstock auf unterschiedlichen Jahren für die Preisbasis." (8)

Internationale Vergleiche der durchschnittlichen Kapitalrentabilität sind deshalb angesichts des Datenproblems bei der Ermittlung der durchschnittlichen Kapitalproduktivität zur Zeit nicht durchführbar. Als Ausweg bietet sich allerdings die Möglichkeit an, die marginale Kapitalrentabilität den internationalen Vergleichen in den Verwertungsbedingungen des Kapitals zugrunde zu legen. Die erforderlichen Daten (Wertzuwachs $\triangle Y$, Nettoanlageinvestitionen I, marginale Lohnquote $\frac{\Delta \mathrm{L}}{\Delta \mathrm{Y}}$ ) sind aus der Volkswirtschaftlichen Gesamtrechnung der verschiedenen Länder leicht zu ermitteln und angesichts des international angeglichenen methodischen Instrumentariums für die Volkswirtschaftliche Gesamtrechnung auch sehr gut vergleichbar.

Im Unterschied zur durchschnittlichen Kapitalrentabilität, die den Teil des gesamten Neuwertproduktes einer Periode auf den gesamten Kapitalstock (Anlagever-

7 Mairesse, Jaques: L'evaluation du Capital fixe productif, les collections de l'lNSEE, C 18-19, Paris 1972, S. 15 ff. und S. 103 ff.

8 Schatz, Klaus-Werner, Wachstum und Strukturwandel der westdeutschen Wirtschaft im internationalen Verbund, Kieler Studien 128, Tübingen 1974, S. 110 
mögen) bezieht, der als Profit von der Bourgeoisie angeeignet wird, setzt die marginale Kapitalrentabilität den von der Kapitalistenklasse angeeigneten zusätzlich in einer Periode produzierten Wert $(\Delta \mathrm{Y})$ in Relation zum Zuwachs des Kapitalstockes in dieser Zeitspanne, den Nettoanlageinvestitionen. Im Zeitablauf lassen sich aus der marginalen Kapitalrentabilität $\Delta r$, sowie ihrer Komponenten, der marginalen Kapitalproduktivität und der marginalen Gewinnquote, Schlüsse auf die Entwicklungsrichtung der durchschnittlichen Kapitalrentabilität und damit der Verwertungsbedingungen des Kapitals ziehen. Sinkt $\Delta \mathrm{r}$ im Zeitablauf kontinuierlich, ist auch $\mathrm{r}$ gefallen, und aus der Entwicklungsrichtung von $\frac{\Delta Y}{I}$ sowie $\left(1-\frac{\Delta L}{\Delta Y}\right)$ lassen sich die wichtigsten Ursachen dieses Trends ablesen. Gegenüber dem Gesamtrentabilitätskonzept hat hierbei das Marginalitätskonzept den Vorteil, Veränderungen in den Rentabilitätsbedingungen schärfer zu akzentuieren. Während bereits leichte Verschiebungen in der Kapitalproduktivität und/oder in den Verteilungsrelationen sehr schnell in der marginalen Kapitalrentabilität sichtbar sind, werden diese Trendveränderungen in den Werten der durchschnittlichen Kapitalrentabilität nur sehr undeutlich dokumentiert.

Aus $\Delta r$ ist aber nicht nur die Entwicklungsrichtung der jeweils nationalen Verwertungsbedingungen ablesbar, ein Vergleich der marginalen Kapitalrentabilität verschiedener Länder läßt auch Rückschlüsse auf die unterschiedlichen Durchschnittsprofitraten im internationalen Vergleich zu. Zwar ist es statistisch durchaus möglich, daß ein Land $A$ in einer Periode von $x$ Jahren stets eine geringere marginale Kapitalrentabilität realisiert als das Land B, obwohl seine absolute Kapitalrentabilität höher liegt als in B; akkumulationstheoretische Überlegungen weisen diese statistische Möglichkeit aber als realiter unwahrscheinlich aus. Das Land A mit einem höheren Akkumulationsniveau und damit einer durchschnittlich geringeren Profitrate wird in der Regel auch eine geringere marginale Kapitalrentabilität verzeichnen als ein weniger entwickeltes Land B. Diese Regel kann in einzelnen Jahren bei einem asynchron verlaufenden Akkumulationszyklus zwischen beiden Ländern durchbrochen werden, je länger aber der beobachtete Zeitraum ist, desto genauer wird $\Delta \mathbf{r}$ nicht nur den Trend, sondern auch das Gefälle in den Verwertungsbedingungen der beiden Länder widerspiegeln (9).

Zur methodischen Diskussion des Indikatorenverfahrens in der Realanalyse vergleiche Ch. Diefenbach u.a.: Wie real ist die Realanalyse? Eine Kritik, sowie Altvater/Hoffmann/ Semmler: Zum Problem der Profitratenberechnung - Eine Replik, in: Prokla Nr. 24, 1976, S. $173 \mathrm{ff}$.

Diefenbach u.a. kritisieren das Rückschlußverfahren der Indikatorenanalyse, die unterstelle, daß ohne Vermittlungen von der Erscheinungsebene (Preisgrößen) auf die Wesensebene (Wertbewegung) zurückgeschlossen werden könne und sich đabei um die Modifikationsanalyse (Monopol, Staat) drücke. M. E. muß jede Realanalyse auf der Basis der Kritik der Politischen Ökonomie mit der Rückschlußhypothese arbeiten, weil per se die Erscheinungsebene nur vermittelter Ausdruck der Wesensebene ist. Auch eine Realanalyse, die auf der Basis einer um die Monopol- und Staatsanalyse erweiterten Ableitung unternommen wird, kommt an der Rückschlußhypothese nicht vorbei. Es ist letztlich eine Differenz in der theoretischen Position und nicht der Verfahrensweise, ob man von der Erscheinungsebene direkt auf die Wesensebene schließt oder zuvor weitere „Zwischen- 


\subsection{Die empirische Struktur der ungleichmäßigen Entwicklung in der EG}

Die Entwicklung der Kapitalakkumulation in Italien, Großbritannien, Frankreich und der BRD von 1950 bis 1975 (vgl. Tabelle 1 und 2) ist durch einen gemeinsamen Grundton gekennzeichnet. Als Ausdruck des Falles der Kapitalprofitabilität, der in allen vier Ländern deutlich zu beobachten ist, sinken auch die Wachstumsraten des Sozialproduktes im längerfristigen Trend. In allen vier Ländern drückt sich der Anstieg der organischen Zusammensetzung des Kapitals in einer immer stärker abfallenden Kapitalproduktivität aus. Gleichzeitig verschiebt sich auch die zweite Komponente der Profitrate, die Profit-Lohn-Relation, im Zeitablauf immer mehr zu Ungunsten des Kapitals. Die Verminderung der Reservearmee, die sich in der BRD, Italien und Frankreich seit Beginn der 60er Jahre deutlich abzeichnet, sowie die Stärkung des ökonomischen Klassenbewußtseins der Arbeiterklasse aller vier Länder seit Ende der 60er Jahre führen zur Aneignung eines größeren Teiles des Wertproduktes durch die Arbeiterklasse. Die stagnativen Tendenzen der Kapitalakkumulation seit Beginn der 70er Jahre sind Resultat sowohl des Absinkens der Kapitalproduktivität als auch der verbesserten Verteilungsposition der Arbeiterklasse.

Wenn auch der ökonomische Grundton in den vier EG-Ländern derselbe ist, verläuft dennoch die Kapitalakkumulation äußerst ungleichmäßig: Die Werte für die Kapitalakkumulation und die Wachstumsraten des Sozialproduktes weichen erheblich voneinander ab.

Eine erste Durchsicht der in den Tabellen 1 und 2 zusammengestellten Daten ergibt folgendes Bild:

a) Italien nimmt in allen Vergleichperioden eine Spitzenposition in der Entwicklung der westeuropäischen Kapitalakkumulation ein. Von 1952 bis 1960 liegt es in den Wachstumsraten des Nettosozialproduktes hinter der BRD an zweiter Stelle, von 1961 bis 1970 übernimmt es die Führungsposition, um von 1971 bis 1974

glieder" einbaut. Ernst zu nehmen ist allerdings das Argument von Diefenbach u.a., daß die im Indikatorenverfahren von Altvater u.a. un terstellte Parallelitätsannahme zwischen Wertgrößen- und Preisgrößenbewegung angesichts der ,relativen Inflation“ sowie der ungleichen Inflationsraten in verschiedenen Sektoren zu problematisieren sei. Die Parallelitätshypothese, die auch in dieser Arbeit unterstellt wird, führt in der Tat zu Vergröberungen und Verfälschungen, die nicht einfach wegdiskutiert werden können. Angesichts zahlreicher ungelöster methodischer Probleme in der marxistischen Inflationsforschung stehen wir zur Zeit vor der Frage, auf empirische Analysen wegen zahlreicher methodischer Mängel ganz zu verzichten oder dennoch - unter Inkaufnahme von Vergröberungen und selbst Fehlern - Ansätze zur Realanalyse zu leisten. In dieser Arbeit ist die Entscheidung zugunsten der empirischen Analyse getroffen worden, weil m.E. der Forschungsfortschritt in der Politischen Ökonomie gerade auch in theoretischen Fragen über die Ergebnisse problematischer Realanalysen eher vorangetrieben wird als im weiteren Beharren auf ,fleischlosen Ableitungen" in der Monopol-, Staats-, Weltmarkt- und Krisenanalyse. Gerade weil die Abstraktionen der Kritik der Politischen Ökonomie beanspruchen, die Wirklichkeit ideell zu reproduzieren und dabei zu entschleiern, können EIkenntnisse über die Notwendigkeit und die Richtung der Verfeinerung unserer Begrifflichkeit erst durch die Versuche einer theoretischen Entschlüsselung der empirischen Verhältnisse gewonnen werden. 
Tabelle $1(10)$

Indikatoren der ungleichmäßigen Entwicklung zwischen Frankreich, Italien, der BRD und Großbritannien von $1952-1974$

$\mathrm{W}_{\mathrm{Y}} \frac{\mathrm{I}}{\mathrm{Y}} \quad \frac{\Delta \mathrm{Y}}{\mathrm{I}} \quad 1-\frac{\Delta \mathrm{L}}{\Delta \mathrm{Y}} \quad \Delta \mathrm{r}$

Frankreich

$\begin{array}{rrrrrr}1952-60 & 4.56 & 9.13 & 0.4840 & 0.4520 & 0.2135 \\ 1961-70 & 5.48 & 13.41 & 0.3948 & 0.3474 & 0.1365 \\ 1971-74 & 4.72 & 15.26 & 0.2919 & 0.2880 & 0.0871\end{array}$

Italien

$\begin{array}{rrrrrr}1952-60 & 5.59 & 12.05 & 0.4466 & 0.4764 & 0.2210 \\ 1961-70 & 5.51 & 14.55 & 0.3597 & 0.3002 & 0.1286 \\ 1971-74 & 3.67 & 12.55 & 0.2764 & -0.2152 & 0.0095\end{array}$

BRD

$\begin{array}{rrrrrr}1952-60 & 7.70 & 16.38 & 0.4319 & 0.4474 & 0.1836 \\ 1961-70 & 4.50 & 17.67 & 0.2392 & 0.2226 & 0.0633 \\ 1971-74 & 2.65 & 15.76 & 0.1532 & 2.3480 & -0.0102\end{array}$

GB

$\begin{array}{rrrrrr}1952-60 & 2.64 & 71.7 & 0.3463 & 0.3397 & 0.1091 \\ 1961-70 & 2.52 & 9.97 & 0.2483 & 0.1152 & 0.0568 \\ 1971-74 & 2.65 & 9.72 & 0.2596 & -1489.4291 & -0.0189\end{array}$

10 Quelle: SAEG: Volkswirtschaftiche Gesamtrechnungen (VGR), 1951 - 1972, 1960 1973, 1960 - 1975, Luxemburg 1973, 1975, 1976; eigene Berechnungen, $\mathrm{W}_{\mathrm{Y}}=$ Wachstumsrate des realen Nettosozialproduktes zu Marktpreisen (1952 - 1960), I bzw. des realen Nettoinlandsproduktes zu Marktpreisen (1961 - 1975);

$\frac{\mathrm{I}}{\mathrm{Y}}=$ Investitionsquote, Nettoanlageinvestitionen dividiert durch $\mathrm{NSP}_{\mathrm{mp}}$ oder $\mathrm{NIP}_{\mathrm{mp}}$ $\frac{\triangle \mathrm{Y}}{\mathrm{I}}=$ marginale Kapitalproduktivität: $\mathrm{Y}_{1}-\mathrm{Y}_{\mathbf{0}}$, dividiert durch $\mathrm{I}_{1}$

$1-\frac{\Delta_{L}}{\Delta Y}$ marginale Gewinnquote; 1 - (Bruttolohnsumme des Jahres 1 minus Bruttolohnsumme des Jahres 0, dividiert durch $\mathrm{Y}_{1}-\mathrm{Y}_{0}$ )

Die Daten von 1952 - 1960 sind zu Preisen von 1963, die Daten von 1961 bis $1965 \mathrm{zu}$ Preisen von 1970 ermittelt. Man beachte, daß die Daten der marginalen Gewinnquote in Zeiten negativer Wachstumsraten einer besonderen Interpretation bedürfen. Werte über 1 signalisieren in Zeiten von Wachstumseinbrüchen eine Umver teilung zugunsten der Arbeiterklasse. Während das Wertprodukt gesunken ist, sind die Löhne weiter angestiegen, so daß die Gewinne stark abfallen. Der Wert der britischen marginalen Gewinnquote im Jahre 1974 von -1490.0 erklärt sich wie folgt: Während das britische Wertprodukt von 1973 auf 1974 von 51.484 Mill. Pfund Sterling auf 51.485 Mill. Pfund Sterling, also um 1 Million angestiegen ist, wuchs die Bruttolohnsumme von 34.609 Mill. Pfund auf 36.100 Mill. Pfund, also um 1.491 Mill. Pfund. Die marginale Lohnquote erreicht deshalb den einmaligen Wert von 1.491 und die marginale Gewinnquote entsprechend: 1 - 1.491 = -1.490 . 
Tabelle 2 (11)

Indikatoren der ungleichmäßigen Entwicklung zwischen Frankreich, Italien, der BRD und Großbritannien von $1952-1975$

$$
\mathrm{W}_{\mathrm{Y}} \quad \frac{\mathrm{I}}{\mathrm{Y}} \quad \frac{\Delta \mathrm{Y}}{\mathrm{I}} \quad 1-\frac{\Delta \mathrm{L}}{\Delta \mathrm{Y}} \quad \Delta \mathrm{r}
$$

Frankreich

$\begin{array}{rrrrrr}1952-55 & 3.98 & 7.17 & 0.5286 & 0.3638 & 0.1851 \\ 1956-60 & 5.03 & 10.70 & 0.4483 & 0.5225 & 0.2362 \\ 1961-65 & 5.89 & 12.14 & 0.4639 & 0.3542 & 0.1615 \\ 1966-70 & 5.06 & 14.68 & 0.3258 & 0.3405 & 0.1115 \\ 1971-73 & 5.38 & 15.60 & 0.3273 & 0.3184 & 0.1039 \\ 1974 & 2.72 & 14.25 & 0.1857 & 0.1967 & 0.0365 \\ 1975 & -1.62 & 13.15 & -0.1249 & 2.4120 & -0.3014\end{array}$

Italien

$\begin{array}{lrrrrr}1952-1955 & 5.66 & 10.51 & 0.5134 & 0.4657 & 0.2593 \\ 1956-60 & 5.53 & 13.29 & 0.3932 & 0.4859 & 0.1903 \\ 1961-65 & 5.00 & 15.69 & 0.2941 & 0.2263 & 0.0852 \\ 1966-70 & 6.01 & 13.41 & 0.4252 & 0.3741 & 0.1719 \\ 1971-73 & 3.75 & 12.52 & 0.2816 & -0.4012 & -0.0171 \\ 1974 & 3.41 & 12.65 & 0.2606 & 0.3427 & 0.0893 \\ 1975 & -3.83 & 10.50 & -0.3788 & 1.3466 & -0.5101\end{array}$

BRD

$\begin{array}{rrrrrr}1952-55 & 9.32 & 15.63 & 0.5466 & 0.4850 & 0.2729 \\ 1956 .-60 & 6.41 & 16.98 & 0.3401 & 0.4173 & 0.1489 \\ 1961-65 & 4.69 & 18.87 & 0.2357 & 0.2494 & 0.0643 \\ 1966-70 & 4.30 & 16.47 & 0.2427 & 0.1957 & 0.0622 \\ 1971-73 & 3.58 & 16.82 & 0.2080 & 0.0289 & 0.0214 \\ 1974 & -0.14 & 12.60 & -0.0113 & 9.3053 & -0.1051 \\ 1975 & -3.24 & 11.15 & -0.3007 & 0.5545 & -0.1667\end{array}$

GB

$\begin{array}{rrrrrr}1952-55 & 2.73 & 6.34 & 0.3962 & 0.2648 & 0.1273 \\ 1956-60 & 2.56 & 7.83 & 0.3064 & 0.3997 & 0.0945 \\ 1961-65 & 3.07 & 9.31 & 0.3168 & 0.1582 & 0.0867 \\ 1966-70 & 1.96 & 10.62 & 0.1797 & 0.0722 & 0.0268 \\ 1971-73 & 3.53 & 9.79 & 0.3460 & .0 .1903 & 0.0741 \\ 1974 & 0.002 & 9.52 & 0.0002 & -1490.0000 & -0.2980 \\ 1975 & -1.91 & 8.95 & -0.2178 & -3.0152 & -0.6568\end{array}$

11 Vgl. Fußnote 10 
hinter Frankreich abermals den zweiten Platz einzunehmen.

Angesichts des Entwicklungsgefälles zwischen Italien und den drei Vergleichsländern überrascht dieses hohe italienische Akkumulationstempo nicht, es bedarf vielmehr umgekehrt einer Erklärung, weshalb die italienischen Wachstumsraten nicht permanent deutlich vor denjenigen Frankreichs, Großbritanniens und der BRD lagen.

b) Frankreich zeichnet sich nach den unterdurchschnittlichen Wachstumsraten in den 50er Jahren seit Beginn der 60er Jahre durch die stabilsten Akkumulationsbedingungen im innereuropäischen Vergleich aus. Die Zuwachsraten des Sozialprodukts, die marginale Kapitalrentabilität, die marginale Kapitalproduktivität sowie die marginale Gewinnquote Frankreichs nehmen seit 1961 europäische Spitzenwerte ein. Neben der bemerkenswerten Steigerung der französischen Wachstumsraten von 1961 bis 1970 gegenüber 1952 bis 1960 bedarf die relative Stabilität der französischen Kapitalakkumulationsbedingungen einer besonderen Erklärung.

c) Großbritannien liegt sowohl in den 50er Jahren wie in den 60er Jahren mit allen Werten für die untersuchten Indikatoren des Akkumulationsprozesses an letzter Stelle. In den 70er Jahren zieht die BRD mit Großbritannien im quantitativen Ausmaß der stagnativen Tendenzen der Kapitalakkumulation allerdings gleich. Die spezielle Akkumulationsstudie über Großbritannien hat die Ursachen zu klären, die die britische Wirtschaft von der europäischen Spitzenposition im Akkumulationsniveau zu Beginn der 50er Jahre durch unterdurchschnittliche Wachstumsraten bis Mitte der 70er Jahre fast auf das italienische Produktionsniveau herabsinken ließen.

d) Der Abwärtstrend, der die europäische Kapitalakkumulation als Ausdruck des tendenziellen Falles der Durchschnittsprofitrate von 1952 bis 1975 kennzeichnet, ist am markantesten in der Bundesrepublik Deutschland ausgeprägt. Von außerordentlich hohen Wachstumsraten in den 50er Jahren sinkt die BRD in den 60er Jahren auf die dritte Position im 4-Länder-Vergleich, um in den 70er Jahren sogar nicht einmal mehr Großbritannien übertreffen zu können. Dieser auffällige Wandel der BRD-Wachstumsraten, der sich im Grunde bereits Mitte der 50er Jahre abzeichnete, verdient in der weiteren Analyse besondere Aufmerksamkeit.

\section{Zu a) Italien:}

Seinem Entwicklungsabstand zu Großbritannien, Frankreich und der BRD entsprechend realisierte Italien in den 50er und 60er Jahren im westeuropäischen Vergleich überdurchschnittliche Kapitalverwertungsbedingungen und damit zusammenhängend überdurchschnittliche Wachstumsraten des NSP. Mit einer marginalen Kapitalrentabilität von durchschnittlich $22 \%$ von 1952 bis 1960 , bzw. $13 \%$ von 1961 bis 1970 sowie Wachstumsraten von durchschnittlich $5.6 \%$ (1952 - 1960) und $5.5 \%$ (1961 - 1970) nahm Italien jeweils die erste oder zweite Position unter den vier westeuropäischen Ländern ein.

Die günstigen Kapitalverwertungsbedingungen waren Resultat eines niedrigen Niveaus in der organischen Zusammensetzung des Kapitals und einer großen industriellen Reservearmee, die die Verteilungsrelationen des Wertprodukts zugunsten des Kapitals verschob. 
$\mathrm{Zu}$ Beginn der 50er Jahre verfügte Italien mit einer Arbeitslosenquote von $10.3 \%$ (12) nicht nur über eine umfangreiche offene Reservearmee, sondern durch überdurchschnittliche Beschäftigungsquoten in weniger produktiven Sektoren auch über eine starke potentielle Arbeitskraftreserve. 1950 lag der Anteil der landwirtschaftlich Beschäftigten an der erwerbstätigen Bevölkerung in Italien bei $42.8 \%$ (zum Vergleich: Frankreich: $29.3 \%$, BRD: $24.8 \%$, Großbritannien: $5.4 \%$ ) (13) und der Anteil der Selbständigen und der Familienbeschäftigten an der nichtlandwirtschaftlich erwerbstätigen Bevölkerung wies in Italien mit $31.3 \%$ ebenfalls einen sehr hohen Wert aus (zum Vergleich: Frankreich: $21.4 \%$, BRD: $15.7 \%$, Großbritannien: $7.7 \%$ ) (14).

Durch die Zerschlagung weniger produktiver Strukturen in Landwirtschaft, Handwerk und Handel konnte das italienische Industriekapital im weiteren Verlaufe des Akkumulationsprozesses billige Arbeitskräfte rekrutieren, deren niedriger Preis gleichzeitig das Tempo des Substitutionsprozesses von Arbeitskraft durch Maschinerie abbremste und so das Wachstum der organischen Zusammensetzung des Kapitals begrenzte. Die marginale Profitquote und die marginale Kapitalproduktivität Italiens lagen in den 50er und 60er Jahren dementsprechend im Vier-LänderVergleich überdurchschnittlich hoch.

Daß sich die Wachstumsraten des Nettosozialprodukts erst in den 60er Jahren und nicht schon in den 50er Jahren an die Spitze in Westeuropa schoben, ist nicht der relativen Verwertungsposition des italienischen Kapitals geschuldet, sondern der im Vergleich zur BRD geringeren Investitionsquote Italiens, die wiederum auf die unterschiedliche Weltmarktintegration beider Länder zurückzuführen ist. Das italienische Kapital wurde in den 50er Jahren im Gegensatz zum BRD-Kapital durch hohe Zollmauern vom Weltmarkt abgeschirmt (15) und erzielte gleichzeitig eine wesentlich geringere Exportquote (Italien: 1952: $9.9 \%$, 1960: 14.7\%; BRD: 1952: $15.8 \%, 1960: 19.9 \%$ ) (16). Der auf das italienische Kapital ausgeubte internationale Konkurrenzdruck war deshalb geringer, so daß trotz günstiger Verwertungsbedingungen die Investitionsquote Italiens mit $12 \%$ in den $50 \mathrm{er}$ Jahren niedriger ausfiel als in der BRD mit $16 \%$.

Durch den Anschluß Italiens an die EG konnte in den 60er Jahren zwar dieses Defizit der italienischen Kapitalakkumulation beseitigt werden und die italienische Wirtschaft jetzt höhere Wachstumsraten realisieren als ihre westeuropäischen Konkurrenten, dennoch bleibt zu klären, weshalb der italienische Wachstumsvorsprung angesichts des großen Produktivitätsrückstandes Italiens nicht erheblich stärker ausgeprägt war.

12 D‘Antonio, Mariano: Sviluppo e crisi del capitalismo italiano 1951 - 1972, Bari 1973, S. 69

13 Denison, E. F., Why Growth Rates Differ, Postwar Experience in Nine Western Countries, Washington D.C., 1967, S. 206

14 Denison, a.a.O., S. 208

15 Kommission der EWG: Bericht über die wirtschaftliche Lage in den Ländern der Gemeinschaft, Brüssel 1958, S. 108

16 Eigene Berechnungen nach: SAEG: VGR, vgl. Fußnote 10 
Die Erklärung für dieses vergleichsweise suboptimale Akkumulationstempo des italienischen Kapitalismus muß m.E. in der unterdurchschnittlichen Entwicklung des italienischen Südens, also im italienischen Nord-Süd-Gefälle gesucht werden. Das Problem der relativen Stagnation des italienischen Südens ist historisch dadurch entstanden, daß im Verlaufe der italienischen Einigungskämpfe der entwickeltere Norden mit dem industriell rückständigen Süden in einem Nationalstaat zusammen geführt wurde. In einer Währungsunion zwangsvereinigt, verlor der weniger entwickelte Süden den Schutzmechanismus in Gestalt von Wechselkursveränderungen, den ansonsten die schwächer entwickelten nationalen Kapitale im Konkurrenzkampf mit höher entwickelten Nationen ausnutzen können (17). Die Ergebnisse des italienischen Einigungskrieges verdammten den relativ rückständigen italienischen Süden zur ökonomischen Stagnation. Bis heute verläuft die Kapitalakkumulation im italienischen Süden relativ langsamer als im Norden und drückt somit das durchschnittliche italienische Akkumulationstempo. Obwohl in den 60er Jahren (1959 bis 1968) im Mezzogiorno $36 \%$ der italienischen Bevölkerung und $30 \%$ der italienischen Erwerbstätigen lebten, wurden hier von 1959 bis 1968 nur $26.7 \%$ der italienischen Bruttoinvestitionen getätigt und nur $23.4 \%$ der Industrieinvestitionen. In der NordOst- und der Zentralregion entsprachen sich Beschäftigungsanteil und Bruttoinvestitionsanteil in etwa, allerdings stiegen auch hier die Industrieinvestitionen unterdurchschnittlich an (18).

In der Konkurrenz zwischen dem Nordwesten und dem Mezzogiorno verfügt der Nordwesten über Infrastruktur- und Marktvorteile, während das niedrigere Lohnniveau im Süden als günstigerer Akkumulationsfaktor zu Buche schlägt. Beim Preisvergleich der Ware Arbeitskraft ist allerdings auch deren Qualifikationsniveau $\mathrm{zu}$ berücksichtigen. So kommt es, daß Industrieunternehmen, die sich im Süden Italiens ansiedeln, häufig einen großen Teil der Belegschaft im Norden rekrutieren, weil für viele industrielle Tätigkeiten das Qualifikationsniveau der gerade proletarisierten Bauern nicht ausreicht. $\mathrm{Zu}$ Ungunsten der Konkurrenzposition des Südens hat es sich in den 60er Jahren, insbesondere seit Ende der 60er Jahre ausgewirkt, daß die italienischen Gewerkschaften eine Politik der Lohnniveauangleichung zwischen dem Norden und dem Süden betrieben und insbesondere in den heißen Arbeitskämpfen seit 1969 verstärkt den Abbau der „Lohnkäfige“ durchgesetzt haben. Heinz-Michael Stahl hat in einer empirischen Untersuchung nachgewiesen, daß im Süden die Lohnzuwachsraten in den 60er Jahren stets über den Produktivitätszuwachsraten lagen und sich damit die Konkurrenzposition des Südens gegenüber dem Norden verschlechterte (19).

Die Unterentwicklung des Südens und die rélative Stagnation des Mezzogiorno, also die Vertiefung der Nord-Süd-Kluft in Italien, beeinträchtigt natürlich die Durchschnittsdaten über das Akkumulationstempo des italienischen Kapitalismus

17 Vergleiche dazu Heinz-Michael Stahl: Regionalpolitische Implikationen einer EWG-Währungsunion, Tübingen 1974, S. 24 ff.

18 Daten aus: Helga Koppel: Klassenkämpfe in Italien heute, Frankfurt a.M. 1972, Tabellen $10-12$, S. 102 ff.

19 Stahl, a.a.O., S. 129 ff. 
beträchtlich. In den ungünstigen Akkumulationsbedingungen des italienischen Südens ist die Hauptursache für das im westeuropäischen Akkumulationspotentialvergleich suboptimale Akkumulationstempo des italienischen Kapitals zu sehen.

\section{zu b) Frankreich:}

Die Wachstumsrate des französischen Wertprodukts belief sich von 1952 bis 1960 auf durchschnittlich 4.6\%. Frankreich lag damit in dieser Periode hinter der BRD $(7.7 \%)$ und Italien (5.6\%) an dritter Stelle im westeuropäischen 4-Länder-Vergleich. Diese unterdurchschnittliche Entwicklung der französischen Kapitalakkumulation in den 50er Jahren läßt sich nicht aus den Verwertungsbedingungen des frảnzösischen Kapitals erklären; diese waren nämlich auch im innereuropäischen Vergleich durchaus günstig. Die marginale Kapitalproduktivität Frankreichs lag von 1952 bis 1960 mit $48 \%$ an der Spitze der vier westeuropäischen Länder, und die marginale Gewinnquote des französischen Kapitals nahm hinter Italien die zweitbeste Position ein. Mit einer durchschnittlichen marginalen Kapitalrentabilität von 21 $\%$ in den 50er Jahren schnitt das französische Kapital im europäischen Vergleich überdurchschnittlich ab. Das hohe Arbeitskräftereservoir in der Landwirtschaft (1950 waren noch $29.3 \%$ aller Erwerbstätigen in der Landwirtschaft beschäftigt) und in Handel, Handwerk und Kleinindustrie (21.4\% aller nichtlandwirtschaftlichen Erwerbstätigen waren 1950 Selbständige oder Familienbeschäftigte) ermöglichte dem französischen Kapital eine günstige Profit-Lohn-Relation und milderte gleichzeitig den Zwang zur Kapitalintensivierung. Nach Berechnungen von Mairesse wuchs die Arbeitsproduktivität (Y/L) der französischen Ökonomie von 1950 bis 1960 mit durchschnittlich $4.9 \%$ schneller als die Kapitalintensität (K/L) mit $3.8 \%$, so daß die Kapitalproduktivität $(\mathrm{Y} / \mathrm{K})$ in dieser Periode um durchschnittlich $1.1 \%$ anstieg (20).

Waren die Verwertungsbedingungen des französischen Kapitals damit unter Berücksichtigung sowohl des Lohnniveaus als auch der Kapitalproduktivität im westeuropäischen Vergleich sehr positiv, so stellt sich die Frage nach den Ursachen des vergleichsweise geringen Wachstums des französischen Wertproduktes in diesem Zeitraum. Die beiden wesentlichen Faktoren der unterdurchschnittlichen französischen Kapitalakkumulation sind m.E. in der protektionistischen Abschirmung Frankreichs vom Weltmarkt bis zur Gründung der EWG und in den ökonomischen Auswirkungen der imperialistischen Politik Frankreichs in Indochina und Algerien zu sehen.

Die französische Industrie wurde in den 50er Jahren durch hohe Schutzzölle, Importkontingentierungen und sonstige nichttarifäre Handelshemmnisse mehr als die Industrie der übrigen entwickelten kapitalistischen Industrieländer von der internationalen Konkurrenz abgeschirmt. Das französische Kapital war in dieser Zeit noch sehr stark auf seine privilegierten Märkte in der Dritten Welt, insbesondere in der Franc-Zone orientiert. 1952 gingen noch $57 \%$ des gesamten französischen Exports in die Länder der Dritten Welt und allein $42 \%$ in die Länder der Franc-Zone.

Mairesse, a.a.O., S. 179 
1960 lag der Anteil der Franc-Zone immerhin noch bei $30 \%$ und die Quote der Dritten Welt insgesamt bei $40 \%(21)$.

Die starke Abschirmung der französischen Wirtschaft vom Druck der Weltmarktkonkurrenz reduzierte für das französische Kapital den Zwang, durch eine forcierte Kapitalakkumulation mit der technologischen Entwicklung in den kapitalistischen Industrieländern Schritt zu halten. Die französische Investitionsquote lag deshalb trotz der sehr guten Verwertungsbedingungen bei nur $7 \%$ von 1952/55 und bei nur $11 \%$ von 1956/60, während Italien und die BRD erheblich höhere Quoten realisieren konnten. Diese relative Stagnation der Investitionen des französischen Kapitals schlug sich trotz der überdurchschnittlichen französischen marginalen Kapitalproduktivität schließlich in den relativ geringen Wachstumsraten des französischen Wertproduktes nieder. Verstärkt wurde diese unterdurchschnittliche Entwicklung der französischen Kapitalakkumulation durch die ökonomischen Auswirkungen der imperialistischen Politik Frankreichs in Afrika und Asien. Der Versuch Frankreichs, die Befreiungsbewegungen in Indochina von 1946 bis 1954 und in Algerien von 1956 bis 1962 mit militärischer Gewalt zu unterdrücken, belastete die französische Wirtschaft sehr stark. Eine hohe Militärlastquote (Anteil der Militärausgaben am BSP) (22) beeinträchtigte die Investitionsquote, und die Finanzierung der Defizite des Staatshaushalts durch zusätzliche Geldproduktion ließ die Inflationsrate ansteigen (23). Bei fester Franc-Parität wurde die Wettbewerbsfähigkeit des französischen Kapitals auf diese Weise durch überdurchschnittliche Inflationsraten und unterdurchschnittliche Produktivitätszuwachsraten unterhöhlt.

Erst die Krise 1957/58 drängte dem französischen Staatsapparat einen radikalen Kurswechsel auf. Der mittels eines Staatsstreichs an die Macht gelangte General de Gaulle vollzog den EWG-Anschluß Frankreichs bei gleichzeitiger Abwertung des Franc und beendete Anfang der 60er Jahre den Algerienkrieg. Der französische Kapitalismus warf damit die beiden wesentlichen Hemmnisse der Kapitalakkumulation der 50er Jahre über Bord und schuf so die Bedingungen für eine neue Prosperitätsphase.

Von der dritten Position in den 50er Jahren schoben sich die Wachstumsraten Frankreichs im Zeitraum von 1961 bis 1970 gemeinsam mit den Zuwachsraten Italiens an die Spitze der vier europäischen Länder. Italien realisierte in den 60er Jahren eine durchschnittliche Wachstumsrate des Nettosozialproduktes von $5.51 \%$ und Frankreich von $5.48 \%$. Gegenüber dem Zeitraum von 1952 bis 1960 mit $4.56 \%$ lagen die französischen Wachstumsraten damit in den 60er Jahren um durchschnittlich jährlich $1 \%$ höher. Diese günstigen Ergebnisse der französischen Kapitalakkumulation von 1961 bis 1970 waren Resultat vor allem zweier Faktoren. Zum einen zwang der EWG-Beitritt und der Abbau des französischen Protektionismus seit 1958 das französische Kapital, aufgrund des schärferen internationalen Konkurrenzkampfes die Investitionstätigkeit zu forcieren, zum zweiten waren die Verwertungs-

21 INSEE: Fresque historique du Systeme productif, E 27, Paris 1974, S. 210

22 A. Glyn/B. Sutcliffe: British Capitalism, Workers and the Profits Squeeze, Harmondsworth 1972 , S. 270

23 Mockers, I.P., Croissances Economiques Comparees, Paris 1969, S. 74 
bedingungen des französischen Kapitals im westeuropäischen Vergleich außerordentlich günstig.

Unter dem Zwang, die Produktivkraft seiner Arbeit angesichts des härteren internationalen Wettbewerbs zu verbessern, steigerte das französische Kapital seine Investitionen in den $60 \mathrm{er}$ Jahren kontinuierlich. Die französische Investitionsquote, die in den 50 er Jahren bei $7.1 \%$ und $10.7 \%$ gelegen hatte, stieg im ersten Jahrfünft der 60er Jahre auf $12.1 \%$ an und im zweiten Jahrfüft auf $14.7 \%$. Diese von der Weltmarktkonkurrenz aufgeherrschte Steigerung des Akkumulationstempos konnte das französische Kapital umso leichter realisieren als seine Verwertungsbedingungen im internationalen Vergleich weiterhin überdurchschnittlich waren. Die marginale Kapitalproduktivität Frankreichs lag in den 60er Jahren mit $39 \%$ weiterhin an der Spitze der vier Vergleichsländer, Italien erreichte $36 \%$, die BRD $24 \%$ und Großbritannien $25 \%$, und auch die marginale Gewinnquote des französischen Kapitals wurde mit $35 \%$ weder von Italien (30\%), noch der BRD (22\%), noch Großbritannien $(12 \%)$ übertroffen. Mit einer Quote von $13.6 \%$ für die marginale Kapitalrentabilität übernahm Frankreich in den 60er Jahren die Spitzenposition unter den vier europäischen Ländern.

Die wesentliche Voraussetzung dieser günstigen Resultate der Kapitalakkumulation Frankreichs war im Vergleich zur Bundesrepublik die günstigere Struktur des französischen Arbeitsmarktes. Frankreich konnte aus der Landwirtschaft sowie aus Handel und Handwerk in den 60er Jahren eine relativ größere Reservearmee für die Industrie mobilisieren als die BRD. Darüberhinaus kehrten von 1960 bis 1969 rund 250.000 Franzosen aus Algerien zurück, die in den französischen Arbeitsmarkt integriert wurden. Schließlich rekrutierte Frankreich in den 60er Jahren insgesamt mehr Gastarbeiter als die BRD: 1968 waren in Frankreich 3.0 Millionen ausländische Arbeiter beschäftigt, das waren ungefähr $15 \%$ der Erwerbstätigen, während in der BRD zum gleichen Zeitpunkt nur 2.3 Millionen Ausländer arbeiteten, die ca. $9 \%$ der Erwerbstätigen ausmachten.

Die günstigeren Arbeitsmarktverhältnisse in Frankreich bewirkten enerseits einen geringeren Lohnquotenanstieg und milderten andererseits den $Z$ wang für das französische Kapital, Arbeitskraft durch Maschinerie zu substituieren, also den Zwang zur Steigerung der technischen Zusammensetzung des Kapitals. Nur so sind die höhere marginale Gewinnquote und die höhere marginale Kapitalproduktivität Frankreichs gegenüber der BRD, die in etwa über das gleiche Akkumulationsniveaư verfügten, zu erklären.

zu c) Großbritannien:

Beim Vergleich der marginalen Kapitalrentabilität und der Wachstumsraten des Nettosozialprodukts der vier EG-Länder fällt im gesamten Zeitraum von 1952 bis 1975 das schlechte Abschneiden des britischen Kapitals auf. Dieses bedarf einer gesonderten Erklärung. Meines Erachtens lassen sich folgende wesentliche Komponenten 
für die relative Stagnation des britischen Kapitalismus anführen (24).

1. Aufgrund des Industrialisierungsvorsprunges, den Großbritannien im 19. Jahrhundert und auch noch zu Beginn des 20. Jahrhunderts gegenüber den übrigen kapitalistischen Industrieländern realisierte, war die Durchkapitalisierung der Landwirtschaft in Großbritannien wesentlich früher abgeschlossen worden als in seinen Konkurrenzländern. So war der Anteil der Landwirtschaft am Sozialprodukt in Großbritannien bereits 1950 auf $3.6 \%$ gesunken, ein Anteil, der nicht einmal bis 1969 von Frankreich und der BRD erreicht wurde und der 1950 auch nicht von den USA $(4.8 \%)$ unterboten wurde $(25)$. Großbritannien verzeichnete damit bereits kurz nach Ende des 2. Weltkrieges ganz im Gegensatz zu seinen europäischen Konkurrenten eine Erschöpfung seines Arbeitskräftereservoirs, einen Arbeitskräftemangel. Die Auswirkungen einer solchen Arbeitsmarktkonstellation auf die Profitquote, die organische Zusammensetzung des Kapitals sowie damit zusammenhängend die Kapitalproduktivität haben wir oben bereits mehrfach dargestellt. Ein Vergleich der marginalen Gewinnquote und der marginalen Kapitalproduktivität zwischen Großbritannien und den drei übrigen EG-Ländern zeigt denn auch, daß Großbritannien bereits in den 50er Jahren eine vergleichsweise geringe Profitquote (33\%) realisierte und die marginale Kapitalproduktivität weit unter den Ergebnissen Frankreichs, Italiens und der BRD lag. Während z.B. das westdeutsche Kapital in den 50er Jahren einen marginalen Kapitalkoeffizienten von 2.3 erreichte, mußte das britische Kapital 2.9 Werteinheiten an Kapital zusätzlich vorschießen, um eine Werteinheit an zusätzlichem Nettosozialprodukt zu produzieren.

2. Von weiterer ausschlaggebender Bedeutung für die relative Stagnation in Großbritannien ist der Wandel in der Weltmarktposition des britischen Kapitals. Nach dem 2. Weltkrieg verlor das britische Kapital durch die Liberalisierung des Welthandels seine Sonderstellung in den Märkten der Commonwealth-Länder und sah sich hier der wachsenden Konkurrenz aus den USA, der BRD, Frankreich, Italien und den übrigen kapitalistischen Industrieländern gegenüber. Zum anderen versuchte das britische Bankkapital trotz der schwindenden Position des britischen Kapitals im Welthandel und der Weltindustrieproduktion und trotz der absoluten Dominanzposition, die das US-Kapital nach dem 2. Weltkrieg erobert hatte, die Rolle der Londoner City als Finanzzentrum der Welt zu erhalten. Dies brachte die frühzeitige Einfuihrung der Währungskonvertibilität (1955), eine Hochzinspolitik und vor allem eine Stabilisierung des überhöhten Außenwerts der britischen Währung (Wechselkurs) mit sich, der im Interesse der Reserveposition des Pfund Sterlings für viele Commonwealth-Länder nicht abgewertet werden durfte. Insbesondere die Überbewertung des Pfund Sterling unterhöhlte die britische Exportposition und produzierte eine chronische Handelsbilanzkrise, wachsende Auslandsverschuldung und den

24 Relative Stagnation soll hier heißen, daß die britischen Wachstumsraten zwar im Vergleich zur Zwischenkriegsperiode durchaus erheblich gestiegen sind, aber im internationalen Vergleich nach 1945 sehr niedrig liegen.

25 Zusammengestellt nach Klaus Werner Schatz: Wachstum und Strukturwandel der westdeutschen Wirtschaft im internationalen Verbund, Tübingen 1974, S. 162 
permanenten Abbau der Währungsreserven (26).

3. Die ungünstigeren Kapitalverwertungsbedingungen im Innern des.Landes und die Überbewertung des Pfund Sterling verfestigten auch nach 1945 die Tendenz des britischen Kapitals zum Kapitalexport. Obwohl das US-Kapital das britische Kapital nach dem 2. Weltkrieg als Hauptkapitalexporteur auf dem Weltmarkt ablöste, lag Großbritannien in der Relation Direktinvestitionen zu Bruttoinvestitionen im Inland weiterhin vor den USA und vor den übrigen kapitalistischen Industrieländern außer der Schweiz. Während die Direktinvestitionen von 1965 bis 1970 in den USA einen Anteil von $4.7 \%$ an den Bruttoinvestitionen erreichten, lag dieser Wert für Großbritannien bei $6.8 \%$ (zum Vergleich: Japan erreichte $1.1 \%$ ) (27). Von 1970 bis 1972 lag der entsprechende Wert für die USA bei $5.1 \%$ und für Großbritannien bei $6.6 \%$, Japan erreichte in diesem Zeitraum $1.5 \%$ (28). Dieses relativ starke Gewicht der britischen Kapitalexporte schwächte natürlich die britische Kapitalakkumulation im Inland um ein weiteres und trug damit ebenfalls zur britischen Stagnation bei.

4. Folge der chronischen Handelsbilanzkrise Großbritanniens unter Nichtantastung des Wechselkurses in den 50er und 60er Jahren war eine häufige Deflationspolitik des britischen Staates, die auf eine Stabilisierung des Außenwerts der Währung orientierte und damit dem Wachstum des britischen Sozialprodukts in der Präferenzskala des Staatsinterventionismus eine geringe Priorität einräumen mußte. Deflationspolitik dominierte in den Jahren 1947, 1949, 1951/52, 1961, 1964 bis 1971 und 1974/75 (29). Die Krise der britischen Kapitalakkumulation wurde auf diese Weise durch die staatliche Wirtschaftspolitik überwiegend verschärft.

5. Seit Ende der 60er Jahre stellt die vergleichsweise starke Kampfposition der britischen Arbeiterklasse einen weiteren restriktiven Faktor furr die britische Kapitalakkumulation dar. Zwar sinken aufgrund des gestiegenen ökonomischen und teilweise auch politischen Bewußtseins der Arbeiterklasse Ende der 60er Jahre in allen wichtigen kapitalistischen Industrieländern die marginalen Gewinnquoten beträchtlich (vgl. Tabelle 2), am schärfsten schlägt sich diese Tendenz aber in Italien und Großbritannien nieder, deren Gewerkschaften in dieser Phase die entschiedensten Kampfpositionen bezogen haben. So ist die durchschnittliche jährliche marginale Gewinnquote von 1971 bis 1974 in Großbritannien negativ, d.h. die Arbeiterklasse hat dem Kapital in dieser Zeit durchschnittlich höhere Lohnzuwächse abgetrotzt als an Neuwert zusätzlich in dieser Periode produziert wurde. Daß der dadurch ausgelöste Druck auf die Durchschnittsprofitrate die britische Kapitalakkumulation eingeschränkt hat, ist selbstverständlich.

26 Pollard; Sidney, The Development of the British Economy 1914 - 1967, London 1976

27 Angaben aus: Dietrich Kebschull/Otto G. Mayer (Hrsg.): Multinationale Unternehmen Anfang oder Ende der Weltwirtschaft?, Frankfurt 1974, S. 23

28 Angaben aus: Henry Krägenau: Internationale Direktinvestitionen 1950 - 1973, Hamburg 1975, S. 43

29 Vergl. Robby Guttmann: Großbritannien - Stagflation und Klassenstrategien, in: Elmar Altvater u.a. (Hrsg.), Handbuch 4 - Inflation - Akkumulation - Krise, 2, Frankfurt a. M. 1976 , S. 138 
$z u$ d) BRD:

Die Analyse des bundesrepublikanischen Akkumulationsprozesses hat zu klären, weshalb die BRD in den 50er Jahren mit durchschnittlich $7.7 \%$ im europäischen Vergleich extrem hohe Wachstumsraten realisierte, aber in den 60er Jahren hinter Italien und Frankreich zurückfiel.

Für die Analyse der Ursachen des außerordentlichen Akkumulationsprozesses in der BRD der 50er Jahre ist es sinnvoll, zwischen den beiden Perioden 1952/55 und 1956/60 zu differenzieren, denn den entscheidenden Wachstumsvorsprung vor seinen westeuropäischen Konkurrenten erreichte das BRD-Kapital mit durchschnittlich $9.3 \%$ in der Zeit von 1952 bis 1955, während in dem darauf folgenden Zeitraum von 1956 bis 1960 die BRD-Wachstumsraten mit 6.4\% diejenigen Frankreichs und Italiens nur noch um durchschnittlich $1.4 \%$ bzw. $0.9 \%$ übertrafen.

In der Periode von 1952 bis 1955 erzielte das BRD-Kapital mit durchschnittlich $27 \%$ die höchste marginale Kapitalrentabilität unter den vier westeuropäischen Ländern. Diese basierte auf der relativ höchsten marginalen Kapitalproduktivität (55\%) und der relativ größten marginalen Gewinnquote (49\%). Bereits in der Zeit von 1956 bis 1960 übertrafen Frankreich und Italien die BRD sowohl in der marginalen Kapitalproduktivität wie auch in der marginalen Gewinnquote.

Für die bacchantischen Akkumulationsbedingungen des BRD-Kapitals im ersten Jahrfünft der 50er Jahre waren hauptsächlich zwei Faktoren verantwortlich:

1. Aufgrund der alliierten Produktionskontrollen über die Westzonen begann die Rekonstruktionsperiode des BRD-Kapitals nach 1945 für westeuropäische Verhältnisse mit einer zeitlichen Verzögerung von etwa 3 Jahren. Während Großbritannien und Frankreich 1949 bereits $123 \%$ der industriellen Produktion von 1938 erreichten und Italien, welches als besiegtes Land ebenfalls alliierten Produktionskontrollen unterworfen war, immerhin schon $104 \%$ des Vergleichsniveaus realisiert hatte, lagen die Westzonen Deutschlands 1949 bei erst $83 \%$ der Industrieproduktion von 1938. Der Kapitalstock (Bruttoanlagevermögen) der Industrie der Bizone, der 1948 mit 23.5 Mrd. Reichsmark (in Werten von 1936) das Bruttoanlagevermögen von 1936 um $11 \%$ überstieg, war 1948 zu nur $50.9 \%$ ausgelastet (30).

Auf der Basis dieses hohen nichtausgelasteten Produktionspotentials konnte das BRD-Kapital seit 1949 überdurchschnittliche Wachstumsraten erzielen. Im Frühjahr 1950 erreichte die Industrieproduktion das Niveau von 1936, wobei das industrielle Bruttoanlagevermögen aber erst zu $73 \%$ ausgelastet war (31). Bis 1955 stieg der industrielle Produktionsindex $(1936=100)$ auf $193 \%$ an $(32)$. Der Auslastungsgrad des industriellen Kapitalstocks entwickelte sich von $73 \% 1950$ über $83.3 \%$ 1953 auf $91.2 \% 1955$ (33).

Die Produktion wuchs folglich in dieser Zeit in ein unausgelastetes Bruttoanlagevermögen hinein, so daß die durchschnittliche Kapitalproduktivität, aber auch

30 Abelshauser, Werner, Wirtschaft in Westdeutschland 1945-1948, Stuttgart 1975, S.114 ff.

31 Kuczynski, Jürgen, Die Geschichte der Lage der Arbeiter unter dem Kapitalismus, Band 7a, Berlin (Ost) 1963, S. 169 und S. 249

32 Kuczynski, a.a.O., S. 247

33 Kuczynski, a.a.O., S. 249 
die marginale Kapitalproduktivität sehr hohe Werte annahmen und äußerst günstige Kapitalverwertungsbedingungen ermöglichten.

2. Die Arbeitsmarktlage bildete die zweite Grundlage der äußerst günstigen Kapitalverwertungsbedingungen anfangs der 50er Jahre. Der Faschismus hatte das Lohnniveau in Deutschland stark gesenkt (34), und die durch die Teilung Deutschlands produzierte hohe industrielle Reservearmee hielt das Lohnwachstum unterhalb des Produktivitätswachstums.

9 Millionen aus Osteuropa vertriebene und geflüchtete Deutsche wanderten in die BRD ein, und bis zum Mauerbau im Jahre 1961 kamen weitere 3 Millionen DDR-Flüchtlinge hinzu.Von diesen 12 Millionen Menschen verkauften etwa 7 Millionen ihre Arbeitskraft auf dem bundesrepublikanischen Arbeitsmarkt, ihr Anteil an der Zahl der Erwerbstätigen betrug damit 1961 fast $27 \%$ (35).

1950 waren in der BRD 1.2 Millionen Menschen arbeitslos, die Arbeitslosenquote betrug $8.2 \% ; 1955$ waren es 495.000 (2.7\%) und 1960 nur noch 130.000 $(0.6 \%)(36)$.

Die hohe industrielle Reservearmee ermöglichte dem bundesrepublikanischen Kapital von 1952 bis 1955 mit $49 \%$ eine marginale Gewinnquote, die weder von Frankreich (36\%) noch von Italien (46\%) übertroffen werden konnte.

Mitte der 50er Jahre waren die außergewöhnlichen Bedingungen, die zu der raschen Kapitalakkumulation in der BRD gefuihrt hatten - der unterausgelastete Kapitalstock und die umfangreichere Reservearmee an Vertriebenen und Flïchtlingen - weitgehend ausgeschöpft. Die Verwertungsbedingungen des BRD-Kapitals unterschieden sich jetzt nicht mehr wesentlich von denen des französischen und italienischen Kapitals. Von 1956 bis 1960 lag die marginale Kapitalrentabilität Frankreichs mit $24 \%$ und die Italiens mit $19 \%$ bereits über der marginalen Kapitalrentabilität der BRD mit $15 \%$.

$\mathrm{Daß}$ das BRD-Kapital trotz eines höheren marginalen Kapitalkoeffizienten als in Frankreich und Italien dennoch etwas höhere Wachstumsraten erzielen konnte, ist auf die wesentlich höhere Investitionsquote der BRD zurückzuführen, die wiederum Ausdruck einer höheren Weltmarktverflechtung der BRD war.

Die Akkumulationsbedingungen des BRD-Kapitals, die bereits beim Übergang vom ersten zum zweiten Jahrfuinft der 50er Jahre eine deutliche Wende verzeichneten, verschlechterten sich in den 60er Jahren aus zwei Gründen:

a) Auf dem Arbeitsmarkt trat eine totale Wende ein: statt eines Arbeitskräfteüberschusses herrschte jetzt Arbeitskraftmangel.

b) Der Zwang zur Substitution von Arbeitskraft durch Maschinerie, der zu einer Steigerung der organischen Zusammensetzung des Kapitals und damit zu sinken-

34 Schoeller/Semmler/Hoffmann/Altvater: Entwicklungstendenzen des Kapitalismus in Westdeutschland (II), in: Prokla 16, S. 63 ff.

35 Kindleberger, C.P., Europe's Postwar Growth, The Role of Labor Supply, Cambridge, Mass. 1967, S. $30 \mathrm{f}$.

36 Welteke, Marianne, Theorie und Praxis der Sozialen Marktwirtschaft, Frankfurt/M. 1976, S. 59 
den Profitraten führt, wurde durch die veränderte Arbeitsmarktlage erheblich verschärft.

$\mathrm{Zu}$ a): Von 1950 bis 1960 wuchs nicht nur die Gesamterwerbstätigenzahl von 20.3 auf 26.2 Millionen -- wobei der Hauptanteil des Zuwachses auf Vertriebene und Flüchtlinge entfiel - es fand auch durch die Zerstörung vor- und kleinkapitalistischer Strukturen in der Landwirtschaft eine erhebliche Landflucht statt, die eine Reservearmee für die Industrie und den tertiären Sektor produzierte. Von 1950 bis 1960 sank die Zahl der landwirtschaftlichen Erwerbspersonen um 1.4 Millionen. Von 1960 bis 1970 wurde in der Landwirtschaft zwar mit 1.3 Millionen etwa dieselbe Zahl an Erwerbspersonen ,freigesetzt" wie in den 50er Jahren, aber die Zahl der Erwerbstätigen erhöhte sich in dieser Periode nur um 500.000. Die Arbeitslosenquote, die bis Mitte der 50er Jahre sehr hoch lag, fiel in den 60er Jahren im Durchschnitt unter $1 \%(37)$.

Dieser radikale Wandel auf dem Arbeitsmarkt verstärkte die objektive Verhandlungsposition der Gewerkschaften und ließ die Löhne jetzt rascher steigen als in den 50er Jahren. Von 1950 bis 1960 nahm die tatsächliche Lohnquote von 58.4 $\%$ auf $60.6 \% \mathrm{zu}$, also um 2.2 Punkte; dagegen wuchs sie bis 1966 allein um 5.1 Punkte auf $65.7 \%$ und bis 1970 - nach dem krisenhaften Absinken im Jahre 1968 - auf $66.7 \%$. Noch deutlicher wird der Wandel zwischen den 50er und 60er Jahren anhand der bereinigten Lohnquote. In den 50er Jahren stiegen die Löhne aufgrund der industriellen Reservearmee langsamer an als die Arbeitsproduktivität, so daß die bereinigte Lohnquote von $58.4 \%$ im Jahre 1950 auf $53.7 \%$ im Jahre 1960 fiel. Umgekehrt in den 60er Jahren: Der Arbeitskraftmangel stärkte die gewerkschaftliche Verhandlungsposition, das Lohnwachstum übertraf das Produktivitätswachstum. Die bereinigte Lohnquote kletterte von $196053.7 \%$ auf $196655.3 \%$, fiel bis 1968 krisenbedingt auf $53.7 \%$ ab, um dann bis 1970 erneut auf $54.8 \%$ anzusteigen (38).

Welche Bedeutung der Arbeitsmarktlage für die Bestimmung der Verteilungsrelationen zukommt, ist auch internationalen Vergleichszahlen zu entnehmen. Obwohl der BRD-Arbeiterklasse kein höheres gewerkschaftliches Kampfbewußtsein bescheinigt werden kann als der französischen und der italienischen Arbeiterklasse, liegt die marginale Gewinnquote in der BRD von 1961 bis 1970 mit $22 \%$ erheblich niedriger als in Italien (30\%) und Frankreich (35\%). Im Vergleich zur BRD konnte die höhere Kampfkraft der italienischen und französischen Arbeiterklasse die Auswirkungen der objektiv schlechteren Verkaufsbedingungen der Arbeitskraft (umfangreichere Reservearmee) auf die Lohnzuwachsraten nicht kompensieren.

$\mathrm{Zu} \mathrm{b):} \mathrm{Die} \mathrm{Tendenz} \mathrm{zur} \mathrm{Substitution} \mathrm{von} \mathrm{Arbeitskraft} \mathrm{durch} \mathrm{Maschinerie,} \mathrm{die}$ in der Kapitalintensität der Produktion indiziert-wird, hat sich mit dem Wandel auf dem Arbeitsmarkt aufgrund der Verknappung und Verteuerung der Ware Arbeitskraft verschärft. Der Konkurrenzkampf des Kapitals, der zur permanenten Verbesserung der Produktionstechnologien zwingt, produziert zwar ohnehin eine Tendenz zur Steigerung der Kapitalintensität, die Arbeitsmarktlage beeinflußt aber eindeutig den Grad der Kapitalintensivierung. So können wir feststellen, daß von 1950 bis

37 Welteke, a.a.O., S. 60

38 Welteke, a.a.O., S. 69; Jahresgutachten 1976/77, a.a.O., S. 68 
1955 die Kapitalintensität in der BRD-Industrie von 53.193 DM Nettokapitalstock je Beschäftigtem auf $195582.823 \mathrm{DM}$ anstieg, also um ungefähr 30.000 DM. Mit den bereits Mitte der 50er Jahre veränderten Akkumulationsbedingungen wurde die Tendenz zur Kapitalintensivierung verschärft: Von 1955 bis 1960 stieg der Nettokapitalstock pro Beschäftigtem um 46.000 DM auf 129.116 DM an. Und schließlich produzierte die Arbeitsmarktlage seit Beginn der 60er Jahre einen neuen Kapitalintensivierungsschub. Bis $1965 \mathrm{nahm}$ die Kapitalintensität um weitere $53.000 \mathrm{DM}$ zu und erreichte insgesamt 182.692 DM (39).

Es zeigt sich, daß die Verwertungsbedingungen in den $60 \mathrm{er}$ Jahren deutlich schlechter wurden. Die marginale Kapitalproduktivität sank von $43 \%$ im Durchschnitt der 50er Jahre auf $24 \%$ von 1961 bis 1970; die marginale Profitquote fiel von $45 \%$ auf $22 \%$ und die marginale Kapitalrentabilität von $18.3 \%$ auf $6.3 \%$.

Daß dieser rasante Abfall der Kapitalrentabilität sich nicht in einem noch stärkeren Wachstumsratenverfall niederschlug, ist allein der hohen Investitionsquote des BRD-Kapitals geschuldet, die bis Mitte der 60er Jahre auf fast $19 \%$ anstieg und auch noch Ende der 60er Jahre mit $16 \%$ über dem Niveau der Investitionsquote Frankreichs und Italiens lag.

Der starke internationale Konkurrenzdruck, der auf dem BRD-Kapital lastete, erzwang trotz sich verschlechternder Verwertungsbedingungen ein hohes Investitionsniveau. Erst mit Beginn der 70er Jahre schlug die weiter absinkende Kapitalrentabilität auch verstärkt auf die Investitionsquote durch.

Die konkrete Form der Weltmarktverflechtung bewahrte die Kapitalakkumulation in der BRD in den 6oer Jahren aus noch einem weiteren Grund vor stärkeren stagnativen Tendenzen.

Die Unterbewertung der DM im Verlaufe der 50er und 60er Jahre ermöglichte den Exportkapitalen der BRD nicht nur äußerst günstige Realisierungsbedingungen auf dem Weltmarkt, sie dämpfte gleichzeitig den Kapitalexport der BRD und ließ umgekehrt den Kapitalimport in die BRD überdurchschnittlich anwachsen. Wegen der unterbewerteten DM exportierten die BRD-Kapitale zu profitablen Bedingungen und waren weniger gezwungen, die Auslandsmärkte durch Direktinvestitionen abzusichern. Umgekehrt exportierte das Ausland in die BRD zu ungünstigeren Bedingungen und sah sich in einem höheren Maße veranlaßt, durch Direktinvestitionen in der BRD die unterbewertete DM zu unterlaufen, um ihre Absatzmärkte in der BRD zu halten.

„Die Bundesrepublik war also lange Zeit ein außerordentlich günstiger industrieller Standort, bedingt vor allem durch die häufige Unterbewertung der DM. Auf der einen Seite schlug sich dies in einer Verzerrung der Kapitalströme nieder, Die Auslandsinvestitionen der westdeutschen Industrie blieben hinter denen vergleichbarer Länder zurück. Die Unternehmen haben sich statt dessen stärker auf die Ausfuhr von im Inland produzierten Gütern konzentriert. Zugleich attrahierte die Bundesrepublik in hohem Maße industrielle Direktinvestitionen aus anderen Ländern, mit denen ein Import modernster Technologie aus dem Ausland einher ging." (40)

Altvater/Hoffmann/Semmler: Zum Problem der Profitratenberechnung, in Prokla 24, S. 206

40 Horn, Ernst-Jürgen, Ist die BRD überindustrialisiert?, in: Wirtschaftsdienst 7/76, S. 347 


\section{Die ungleichmäßige Entwicklung der Inflation in den kapitalistischen Indu- strieländern}

Die Inflationsanalyse ist in diesem Abschnitt wie folgt angelegt: Zunächst wird nach den Ursachen des trendmäßig sich verschärfenden Anstieges des allgemeinen Preisniveaus seit Beginn der 60er Jahre gefragt, also nach Erklärungen für die sich trendmäßig verschärfende schleichende Inflation gesucht. In einem zweiten Schritt werden dann die ungleichen Inflationsraten in den kapitalistischen Industrieländern, speziell in der EG, analysiert und Hypothesen für die Dechiffrierung dieses Phänomens diskutiert.

\subsection{Zur Theorie der permanenten Inflation}

Eine Theorie der permanenten Inflation in den kapitalistischen Industrieländern seit Ende des 2. Weltkrieges hat vor allem zwei Fragen zu beantworten. Zum ersten hat sie über die Tatsache Aufschluß zu geben, daß das Preisniveau in Krisenzeiten gegenüber Boomperioden nicht nur nicht mehr absinkt, wie es für die Zyklen bis zur groBen Weltwirtschaftskrise noch der Fall war, sondern sogar noch rascher ansteigt als in Zeiten guter Konjunktur. Zweitens muß eine Inflationstheorie erklären, welche Faktoren für den trendmäßigen Anstieg der Inflationsraten seit Beginn der 50er Jahre verantwortlich sind.

Zu 1: Nach den Keynes verpflichteten Inflationstheorien, die die Steigerung des allgemeinen Preisniveaus aus einem Überhang der gesamtwirtschaftlichen Nachfrage gegenüber dem gesamtwirtschaftlichen Angebot erklären, müßten im Verlaufe des Konjunkturzyklus Preissteigerungen in den Aufschwungs- und Boomperioden und Preisnachlässe in den Abschwüngen und Krisenphasen des Zyklus zu beobachten

Tabelle 3 (41)

Die inverse Beziehung zwischen Inflationsraten und Wachstumsraten

B R D Frankreich Großbritannien

Jahre mit den höchsten

Preissteigerungsraten

Jahre mit den geringsten Wachstumsraten des Bruttoinland sproduktes
$51,52,57,58,62 \quad 51,52,58$

$57,58,62,63$

$52,53,58,59$
$51,52,55,56,57$, $58,62,65$

$51,52,55,56,57$, $58,62,65$ 
sein. Tatsächlich läßt sich aber im Inflationsrhythmus seit Beginn der 50er Jahre genau die gegenteilige Tendenz beobachten. Wider Erwarten steigen die Preise in Zeiten geringeren wirtschaftlichen Wachstums und in Krisenzeiten schneller an als in den Phasen günstiger Konjunktur. Mockers hat den Zusammenhang von Wachstum und Preissteigerungsraten in den drei Ländern BRD, Frankreich und Großbritannien von 1950 bis 1965 untersucht. Seine Ergebnisse sind in der Tabelle 3 wiedergegeben.

In der Mehrzahl der von ihm untersuchten Fälle stellt Mockers fest, daß die Preissteigerungsraten gerade in den Jahren am höchsten sind, in denen das Bruttosozialprodukt mit den geringsten Raten wächst. Diese inverse Beziehung von Wachstumsraten und Inflationsraten beobachtete Mockers ebenfalls in den USA, Schweden, Italien .und den Niederlanden. In zwei von drei Fällen korrespondierte in diesen Ländern nach Mockers ein Absinken der Wachstumsraten mit einem Anstieg des allgemeinen Preisniveaus (42).

Wie Mockers, so konnte auch Pollard in seiner Analyse der britischen Wirtschaftsentwicklung feststellen, daß die Preise in den Stagnationsphasen Großbritanniens stärker anstiegen als in den Expansionsperioden. In den Stagnationsphasen (1955 - 58, 1960 - 62, 1964 - 66) lagen nach Pollard die jährlichen Wachstumsraten der industriellen Produktion bei $0.9 \%$, die Steigerungsraten der Einzelhandelspreise aber bei $4.0 \%$ (Preissteigerungsraten der verarbeitenden Industrie $2.9 \%$ ). Umgekehrt in den Expansionsperioden $(1953$ - 55, 1958 - 60, 1962 - 64): Während die industrielle Produktion um durchschnittlich jährlich $5.8 \%$ wuchs, stiegen die Einzelhandelspreise um $2.1 \%$ an und die Preise der verarbeitenden Industrie um 1.4 $\%$ (43).

In Italien läßt sich dieses inverse Verhältnis von Wachstumsraten und Preissteigerungsraten von 1952 bis 1965 ebenfalls beobachten. In den Jahren rückläufiger Wach stumsraten des Nettosozialproduktes, 1952, 1954, 1956, 1958, 1962 - 65, ist mit Ausnahme des Jahres 1954 die Inflationsrate angestiegen. Am deutlichsten zeigt sich dieses Phänomen in der Krise 1964/65 und ihrer Vorphase 1962/63. Gegenüber dem Durchschnitt der Jahre 1952 bis 1961 verdoppelte sich von 1962 bis 1965 die Inflationsrate (44).

Da diese inverse Beziehung zwischen Preissteigerungsraten und Wachstumsraten des Sozialprodukts mit der gesamtwirtschaftlichen Nachfragetheorie nicht erklärt werden kann, stellt sich die Frage nach alternativen Erklärungsmodellen. Ausgehend von den hohen Inflationsraten in den kapitalistischen Industrieländern während der Weltmarktkrise 1974/75 begründen Altvater u.a. die höheren Inflationsraten in der Krise aus der relativ gewachsenen Bedeutung des fixen Kapitalanteils am insgesamt vorgeschossenen produktiven Kapital, die dazu führe, daß bei unterausgelasteten Kapazitäten in der Krise die Kostenbelastung für die Kapitale enorm anwachse und so Preiserhöhungen erzwinge.

42 Mockers, a.a.O., S. 212

43 Pollard, a.a.o., S. 483

44 Podbielski, Gisele, Italy: Development and Crisis in the Post-War Economy, Oxford 1974, S. 103 
„Daraus ergibt sich für jedes Einzelkapital der ökonomisch bedingte Zwang, gerade in der Krise bei Absatzstockungen und Unterauslastungen der Produktionskapazitäten, keine Preissenkungen vorzunehmen. Vielmehr wird das jeweilige Einzelkapital eher versuchen, seine Preise heraufzusetzen infolge der gestiegenen Kostpreise. Gerade in der Krise ist hier eine materielle Basis dafür gegeben, daß die Preise steigen anstatt zu sinken." (45)

Das Phänomen steigender Kostpreise aufgrund wachsender Fixkostenbelastungen bei unterausgelasteten Kapazitäten ist sicherlich nicht auf die Krisen seit Ende des 2. Weltkrieges oder seit Mitte der 70er Jahre zu beschränken, sondern bereits - wenn auch nicht im selben relativen Ausmaße - in allen Krisenperioden im 19. Jahrhundert zu beobachten. Insofern stellt sich beim Erklärungsansatz: von Altvater u.a. zum einen die Frage, welche Momente bis zur Weltwirtschaftskrise der 30er Jahre die Preise in der Krise trotz angestiegener Kostpreise fallen ließen, seit Beginn der 50er Jahre aber die umgekehrte Tendenz produzierten. Zum zweiten wird bei Altvater u. a. - da sie vollständige Konkurrenz unterstellen - der Transmissionshebel nicht deutlich, der bei sinkender Nachfrage dem Kapital dennoch das Durchsetzen höherer Preise ermöglicht. Demele bemerkt mit Recht:

„Von den Autoren müßte daher gezeigt werden, wie sich Einzelkapitale unter Konkurrenzbedingungen, d.h. bei grund sätzlicher Gültigkeit des Gesetzes von Angebot und Nachfrage, zu dem Versuch entschließen können, die Preise ihrer Waren zu erhöhen, obwohl die Nachfrage nicht nur nicht gestiegen, sondern (in der Krise) sogar gesunken ist.

Ohne ausreichende Marktmacht würde jedes Einzelkapital, das zum Zwecke der Preisanhebung z. B. das eigene Angebot über das von der Nachfrageseite her ohnehin erzwungene Maßs hinaus reduzieren wollte, Gefahr laufen, daß seine Konkurrenten sofort in die entstehende Angebotslücke mit den ,alten“ Preisen stoßen würden, um über einen steigenden mengenmäßigen Absatz ihre Marktanteile und vor allem ihre Profite zu erhöhen." (46)

Eine widerspruchsfreie Erklärung der inversen Beziehung von Wachstumsraten und Inflationsraten ist von daher nur möglich, wenn man die Prämisse vollständiger Konkurrenz für die Kapitalakkumulation seit 1945 aufgibt und die Existenz oligopolistischer Marktstrukturen unterstellt. Erst wenn man von einer Preisfestsetzungsmacht in zahlreichen Branchen der kapitalistischen Industrieländer seit Ende des 2. Weltkrieges ausgeht, wird das Phänomen steigender Inflationsraten in den Krisenperioden der Kapitalakkumulation verständlich. Nur auf der Basis eingeschränkter Preiskonkurrenz ist es möglich, daß das Kapital in der Krise bei steigenden Stückkostenbelastungen über hohe Preise den Versuch wagen kann, den Profitratenabfall zu stoppen. Bei vollständiger Konkurrenz würde ein Einzelkapital, das die Preise bei sinkender Nachfrage nach oben setzt, das Risiko eingehen, daß ihm seine Konkurrenten auf der Basis der alten Preise Marktanteile abjagen, um so ihren Profitratenabfall teilweise auffangen zu können.

$\mathrm{Zu}$ 2: Lassen sich aus den relativ gewachsenen Kostenbelastungen des Kapitals bei oligopolistischen Marktverhältnissen die vergleichsweise hohen Inflationsraten der kapitalistischen Industrieländer in der Krise widerspruchsfrei deuten, ist damit das

45 Altvater/Hoffmann/Künzel/Semmler: Krise und Inflation, in: Handbuch 3, Inflation Akkumulation - Krise, I, Frankfurt/Main 1976, S. 128

46 Demele, Ottwald, Die Rolle großer Konzerne im Inflationsprozeß, zur Diskussion um Preissteigerungen in der Krise, in: Prokla 25, S. 136 
Problem der permanenten Inflationsraten und die Frage der trendmäßig wachsenden Inflationsraten seit Beginn der 60er Jahre noch nicht geklärt. Aus der Tabelle 4 geht der Trend der Inflationsraten in den kapitalistischen Industrieländern sehr

Tabelle 4 (47)

Die Entwicklung der Weltinflation

\begin{tabular}{|c|c|c|c|c|}
\hline $\begin{array}{l}\text { Ausgewählte industrialisierte } \\
\text { Länder }\end{array}$ & $\begin{array}{l}1953 \\
1955\end{array}$ & $\begin{array}{l}1.956 \\
1965\end{array}$ & $\begin{array}{l}1966 \\
1972\end{array}$ & $\begin{array}{l}1973 \\
1974\end{array}$ \\
\hline 1. USA & 0.4 & 1.6 & 4.1 & 8.6 \\
\hline 2. Großbritannien & 3.0 & 3.4 & 5.6 & 12.6 \\
\hline 3. Bundesrepublik & 0.0 & 2.3 & 3.5 & 7.0 \\
\hline 4. Frankreich & -0.2 & 3.2 & 4.8 & 10.4 \\
\hline 5. Italien & 2.2 & 3.6 & 3.6 & 15.0 \\
\hline 6. Kanada & 0.1 & 1.8 & 3.8 & 9.3 \\
\hline 7. Japan & 3.0 & 3.9 & 5.4 & 13.1 \\
\hline \multicolumn{5}{|l|}{ 8. Industrialisierte Länder } \\
\hline insgesamt* & 0.7 & 2.3 & 4.4 & 10.5 \\
\hline
\end{tabular}

* = OECD-Länder plus Australien, Neuseeland, Südafrika und Israel, gewogen mit dem Anteil der Länder am gesamten BIP.

deutlich hervor. Die gewogene.durchschnittliche Inflationsrate der kapitalistischen Industrieländer stieg von 1956/65 $2.3 \%$ auf 1966/72 4.4\%, um 1973/74 10.5\% zu erreichen. Was für den Durchschnitt gilt, spiegelt sich in den Zahlen für die einzelnen Länder wider. Ausnahmslos hat sich in allen in der Tabelle angeführten kapitalistischen Industrieländern der Inflationstrend seit Mitte der 50er Jahre permanent verschärft.

Nach der obigen Analyse der Kapitalakkumulation in ausgewählten EG-Ländern von 1952 bis 1975 drängt sich für die Erklärung der permanenten und trendmäßig ansteigenden Inflation folgende Hypothese auf. Die Preissteigerungen sind der Versuch des Kapitals, auf der Basis vermachteter Märkte die Profitrate durch Umverteilungen zwischen der Arbeiterklasse und der Bourgeoisie einerseits und zwischen den verschiedenen Anlagesphären des Kapitals andererseits zu verbessern. Diese Profitratensanierungsversuche des Kapitals über die Inflation müssen in dem MaBe an Gewicht gewinnen, wie sich durch die Steigerung der organischen Kapitalzusammensetzung und die Verschiebung in der Profit-Lohn-Zusammensetzung zugunsten der Löhne ein trendmäßiger Abfall in der Kapitalrentabilität bemerkbar macht. Der permanente Anstieg der Inflationsraten wäre damit nichts anderes als das Spiegelbild des tendenziellen Falles der Profitrate in einer Zeit zunehmend organisierter Märkte.

47 Siebke, Jürgen/Zumpfort, Wolf-Dieter, Weltinflation und Inflationstheorien, in: Wirtschaftsdienst, Nr. 10/75, S. 524 
Den Zusammenhang zwischen dem Anstieg der Inflationsrate und dem Fall der Kapitalrentabilität für sechs kapitalistische Industrieländer veranschaulicht die Tabelle 5. Für alle in der Tabelle aufgeführten Länder läßt sich ein eindeutiger $\mathrm{Zu}$ sammenhang zwischen einer Verschlechterung der Kapitalverwertungsbedingungen

Tabelle 5 (48)

Die Entwicklung der marginalen Kapitalrentabilität und der Inflationsrate in ausgewählten kapitalistischen Industrieländern $1961-1975$

\begin{tabular}{llrrr} 
& & $1961-$ & $1966-$ & $1971-$ \\
& & 1965 & 1970 & 1975 \\
\hline USA: & $\Delta \mathrm{r}$ : & 26.5 & -0.5 & -4.6 \\
& Inflationsrate: & 6.0 & 18.4 & 40.1 \\
BRD: & $\Delta \mathrm{r}$ : & 6.4 & 6.2 & -4.1 \\
& Inflationsrate: & 13.6 & 15.6 & 38.0 \\
Frankreich: & $\Delta \mathrm{r}$ : & 16.1 & 11.1 & 1.0 \\
Großbritannien: & Inflationsrate: & 15.4 & 19.3 & 55.6 \\
& $\Delta \mathrm{r}$ : & 8.6 & 2.6 & -14.6 \\
Italien: & Inflationsrate: & 12.4 & 21.1 & 90.5 \\
& $\Delta \mathrm{r}:$ & 8.5 & 17.2 & -47.0 \\
Japan: & Inflationsrate: & 19.9 & 15.6 & 82.0 \\
& $\Delta \mathrm{r}:$ & 17.2 & 24.7 & $1.4 *$ \\
& Inflationsrate: & 16.0 & 23.0 & 63.6 \\
\hline
\end{tabular}

$\triangle \mathrm{r}$ : Durchschnittliche Jahreswerte der Perioden

Inflationsrate: Preisindex der letzten Inland sverwendung, 1970:100, * = $1971-1974$

und einem Anstieg der Inflationsrate festmachen, wobei die Inflation in der relativen Stagnationsphase seit 1970 am stärksten angestiegen ist. Der gegenläufige Trend, der sich in sinkenden Inflationsraten bei sich verbessernder Kapitalrentabilität bemerkbar machen müßte, ist zwar in Italien im Periodenvergleich 1966/70 gegenüber 1961/65 deutlich zu erkennen, hingegen nicht bei Japan.

Man kann die empirische Basis noch dadurch erweitern, daß der Ausgangspunkt der Betrachtung auf Mitte der 50er Jahre - nach dem Auslaufen der Koreainflation und dem Abschluß der Rekonstruktionsperiode in allen kapitalistischen Industrieländern - zurück verlegt wird. Tabelle 6 zeigt den hier behaupteten Zusammenhang von veränderten Verwertungsbedingungen und Inflationsraten deutlich an. In den USA sinken die Inflationsraten von 1961/64 gegenüber 1957/60, weil sich die Kapitalrentabilität verbessert, in der BRD, Großbritannien, Italien und in Japan steigen die Inflationsraten parallel $\mathrm{zu}$ sich verschlechternden Verwertungsbedingungen. Nur Frankreich verzeichnet 1961/64 gegenüber 1957/60 einen Fall der Inflationsrate trotz sich verschlechternder Kapitalrentabilität. Bei den sehr hohen 
französischen Inflationsraten seit Mitte der 50er Jahre muß die Auswirkung des Algerienkrieges auf die französische Wirtschaft berücksichtigt werden. Der Kolonialkrieg in Algerien wurde angesichts der Defizite des französischen Staatshaushaltes

Tabelle 6 (49)

Die Entwicklung der marginalen Kapitalrentabilität und der Inflationsrate in ausgewählten kapitalistischen Industrieländern $1957-1964$

\begin{tabular}{llrr}
\hline & & $1957-1960$ & $1961-1964$ \\
\hline USA & $\triangle \mathrm{r}:$ & 3.0 & 26.5 \\
& Inflationsrate: & 8.8 & 5.3 \\
BRD: & $\triangle \mathrm{r}:$ & 14.0 & 6.0 \\
& Inflationsrate: & 8.7 & 13.4 \\
Frankreich: & $\Delta \mathrm{r}:$ & 23.0 & 16.0 \\
& Inflationsrate: & 20.2 & 16.9 \\
Großbritannien: & $\Delta \mathrm{r}:$ & 9.0 & 8.0 \\
& Inflationsrate: & 8.7 & 12.1 \\
Italien: & $\triangle \mathrm{r}:$ & 19.0 & 8.0 \\
Japan: & Inflationsrate: & 5.0 & 21.6 \\
& $\triangle \mathrm{r}:$ & 24.0 & 17.2 \\
& Inflationsrate: & 9.6 & 17.9 \\
\hline
\end{tabular}

$\Delta \mathrm{r}$ : Durchschnittliche Jahreswerte der Perioden $1956-60,1961-65$

Inflationsrate: Preisindex der letzten Inlandsverwertung, $1963=100$

teilweise über die direkte Geldproduktion der französischen Zentralbank finanziert und bewirkte von daher eine starke zusätzliche Inflationierung der französischen Wirtschaft.

Insgesamt können wir angesichts des Vergleiches von Kapitalverwertungsveränderungen und Inflationsraten von 1957 bis 1975 festhalten, daß für die USA, die $B R D$, Italien und Großbritannien in allen Periodenvergleichen eine eindeutig inverse Beziehung zwischen Inflationsraten und den Veränderungen der Kapitalrentabilität besteht. Bei Frankreich und Japan konnte beim Vergleich beider Indikatoren in vier Perioden nur jeweils eine Abweichung festgestellt werden.

\subsection{Zur Erklärung der ungleichmäßigen Entwicklung der Inflation in den kapitali- stischen Industrieländern}

Gehen wir jetzt dazu über, die ungleichen Inflationsraten zwischen den kapitalistischen Industrieländern zu untersuchen, dann können wir zunächst - obwohl es nach den bisherigen Ausführungen über die Ursachen der sich trendmäßig verschärfenden

49 Quelle: SAEG: VGR, Vgl. Fußnote 10 
Inflation zu erwarten wäre - keinen direkten Zusammenhang zwischen den Abweichungen in der Kapitalrentabilität der einzelnen Länder und den Niveauunterschieden in ihren Inflationsraten beobachten. Die Länder mit den günstigsten Kapitalverwertungsbedingungen verzeichnen nicht die geringsten Inflationsraten, ja, für die 60er Jahre ist sogar die gegenteilige These richtig. Von 1961 bis 1970 liegen in den Ländern mit einer höheren marginalen Kapitalrentabilität, Japan, Frankreich und

Tabelle 7 (50)

Price Rise by Sectors (1958 - 1968 at annual rates)

\begin{tabular}{lccccc}
\hline Country & Agriculture & Industry & Buildings & Services & GDP \\
\hline & & \multicolumn{3}{c}{ Price rise ${ }^{2}$} \\
United States & 0.9 & 0.9 & 5.2 & 2.6 & 2.1 \\
United Kingdom & -0.3 & 2.2 & 3.5 & 4.5 & 2.8 \\
France 3 & 2,6 & 2.4 & 4.8 & 5.1 & 3.9 \\
Germany & 0.1 & 1.7 & 4.4 & 4.2 & 2.8 \\
Italy & 1.9 & 1.8 & 6.1 & 5.1 & 3.7 \\
Netherlands & 1.7 & $2.6^{4}$ & - & 4.9 & 3.7 \\
& & & & & \\
& & & Weight in GDP & & \\
United States & 4.6 & 32.4 & 4.6 & 58.1 & 100 \\
United Kingdom & 4.3 & 41.0 & 5.9 & 47.2 & \\
France & 9.2 & 40.1 & 8.0 & 42.7 & \\
Germany & 6.5 & 47.0 & 6.3 & 40.0 & \\
Italy & 18.5 & 28.6 & 7.3 & 45.6 & \\
Netherlands & 11.2 & $41.3^{4}$ & - & 47.5 & \\
\hline
\end{tabular}

$1=$ Mining, manufacturing and public utilities

$2=$ Change of implicit price deflator of the value-added of each sector

$3=1959-1968$

4 = Including construction

Italien, die Inflationsraten über den Preissteigerungsraten der Länder mit niedriger marginaler Kapitalrentabilität, der BRD und Großbritannien. Was also für den Verlauf der Inflationsraten innerhalb der einzelnen Länder gilt, daß sich ein niedriges Niveau in höheren Inflationsraten niederschlägt und umgekehrt, läßt sich für den Inflationsratenvergleich zwischen verschiedenen Ländern nicht feststellen. Offensichtlich bedarf das ungleiche Niveau der Inflationsraten zwischen verschiedenen kapitalistischen Industrieländern einer zusätzlichen Erklärung.

M. E. muß zur Ableitung der ungleichen nationalen Inflationsraten das ungleiche sektorale nationale Produktivitätsgefüge zwischen verschieden entwickelten Ländern mit herangezogen werden. Ehe diese Hypothese vollständig dargestellt werden

50 Quelle: OECD: Inflation, the present problem, Paris 1970, S. 61 
kann, sollen zunächst die Inflationsraten der verschiedenen Sektoren der Ökonomie in den einzelnen Ländern näher beleuchtet werden. (Vgl. die Tabellen 7 und 8) Die beiden Tabellen über den Preisanstieg in den Sektoren Landwirtschaft, Industrie und Dienstleistungen sowie den Preisanstieg der verschiedenen Komponenten der Verbraucherpreise zeigen sehr deutlich, daß sich die Preise des Industriesektors (bei den Verbraucherpreisen die Komponente „Other goods“) in allen Ländern unterdurchschnittlich entwickelt haben, während die Inflationsraten des Dienstleistungssektors weit über dem Durchschnitt lagen. Diese Preisschere zwischen dem Industrieund dem Dienstleistungssektor ist aus der Produktivitätsschere zwischen beiden Sektoren abzuleiten. Das Industriekapital inflationiert seine Waren angesichts seiner überdurchschnittlichen Produktivitätsfortschritte nur in geringem Maße absolut, aber im hohen Maße relativ, da es die sinkenden Werte pro Einzelware nicht in Preisnachlässen weitergibt (51). Angesichts seiner unterdurchschnittlichen Produktivitätsfortschritte ist das Dienstleistungskapital dagegen zu höheren absoluten Preissteigerungen gezwungen, will es sich weiterhin den gesellschaftlichen Durchschnittsprofit aneignen.

Dieser Preissteigerungszwang vermittelt sich dem Dienstleistungskapital iber die leicht gestiegenen Preise der Inputs aus Industriewaren, vor allem aber angesichts Tabelle 8 (52)

Consumer Prices by Standard Groups, Average annual rates of change, $1960-1969$

\begin{tabular}{llllll}
\hline Country & Food & Other goods & Rent & Other Services & Total \\
\hline United States & 2.6 & 1.7 & 1.6 & 3.6 & 2.4 \\
Canada & 2.6 & 1.4 & 3.4 & 4.2 & 2.7 \\
Japan & 6.2 & 3.1 & 8.2 & 7.2 & 5.5 \\
United Kingdom & 3.8 & 3.2 & 5.1 & 5.1 & 4.0 \\
France & 3.9 & 2.6 & 8.9 & 5.9 & 3.9 \\
Germany & 2.0 & 1.6 & 6.4 & 4.0 & 2.6 \\
Italy & 3.4 & 2.8 & 6.3 & 6.1 & 3.8 \\
Netherlands & 4.2 & 3.1 & 4.9 & 5.4 & 4.2 \\
\hline
\end{tabular}

der großen Arbeitsintensität der Dienstleistungen über die Lohnsteigerungsraten, die sich im stärkeren Maße an den überdurchschnittlichen Produktivitätszuwächsen im Industriesektor orientieren. Da die Produktivitätsfortschritte mit dem Kostenwachstum nicht Schritt halten, sind Preissteigerungen notwendig, soll die Profitrate nicht unter den gesellschaftlichen Durchschnitt absinken (53). Die Inflationsraten des Dienstleistungssektors werden dabei umso höher sein, - je größer der Produktivitätsabstand zum Industriesektor ist,

51 Zum Problem der relativen Inflation vgl.: Hofmann, Werner, Die säkulare Inflation, Berlin o.J., S. 10

52 Quelle: OECD: Inflation, The present problem, Paris 1970, S. 61

53 Zum Problem unterschiedlicher Inflationsraten bei ungleichen Produktivitätsfortschrittẹn, vgl. auch Demele, a.a.O., S. 148 ff. 
- je stärker die Lohnzuwachsraten ausfallen,

- je weniger der Industriesektor seine Produktivitätsfortschritte in Preisnachlässen weitergibt, je stärker dieser Sektor also seine Waren relativ inflationiert.

Interessanterweise gehen damit die größten absoluten Preissteigerungen nicht vom hochkonzentrierten und hochzentralisierten Industriesektor aus, sondern von einem ökonomischen Sektor, der unterdurchschnittlich zentralisiert ist, eine geringe organische Zusammensetzung des Kapitals aufweist und unterdurchschnittliche Produktivitätszuwachsraten realisiert.

Versuchen wir diese Ausführungen über die sektoralen Preisdifferenzen mit der allgemeinen Inflationserklärung zu verbinden, dann können wir feststellen: $U$ sache der permanenten Inflation, die sich trendmäßig verschärft, ist der Versuch des Kapitals, der Verschlechterung der Verwertungsbedingungen durch Preissteigerungen entgegenzuwirken. Voraussetzung der permanenten Inflation ist eine oligopolistische Struktur der Märkte, die die Preiskonkurrenz einschränkt. Das Ausmaß der Inflationsraten hängt ab a) vom Grad des Profitratenfalles, b) vom Grad der sektoralen Inhomogenität der nationalen Ökonomie.

Bei der Untersuchung der Inflationsraten in ungleich entwickelten Nationen ist vor diesem Hintergrund zu berücksichtigen, daß die Homogenität der sektoralen Struktur der Ökonomie in weniger entwickelten Ländern geringer ist als in hoch entwickelten kapitalistischen Industrieländern. Diese größere Inhomogenität bezieht sich dabei nicht nur auf das Verhältnis vom Industriesektor zum Dienstleistungssektor, sondern ebenfalls auf das Produktivitätsgefälle zwischen dem Agrarsektor und den beiden übrigen Sektoren der Gesamtwirtschaft sowie auf die Differenzierungen innerhalb der drei Hauptsektoren selbst. Wenn es richtig ist, daß sich im Zuge der Durchkapitalisierung eines Landes zunächst die Produktivitätsschere zwischen dem Industriesektor auf der einen Seite und dem Agrar- und dem Dienstleistungssektor auf der anderen Seite öffnet und sich auch die Produktivitätsabstände zwischen den verschiedenen Zweigen des Industriesektors im Verlaufe des Entwicklungsprozesses zunächst vergrößern, in einer späteren Entwicklungsphase auf einem höheren absoluten Akkumulationsniveau diese Produktivitätsscheren aber wegen der überdurchschnittlichen Akkumulation in den unterdurchschnittlich produktiven Sektoren nach und nach verringert werden, dann wären aufgrund dieser sektoralen Strukturdifferenzen die Inflationsraten in den schwächer entwickelten Ländern höher als in den entwickelteren Nationen.

Aus den Tabellen 7 und 8 ist nicht nur deutlich abzulesen, daß die Inflationsraten des Dienstleistungssektors über den Preissteigerungsraten des Industriesektors liegen, sondern ebenso klar erkennbar, daß der Abstand zwischen den höher entwickelten Ländern (USA, Kanada, BRD) und den weniger entwickelten Nationen (Japan, Italien) in den Inflationsraten des Industriesektors (bzw. bei den Verbraucherpreisen in den Preissteigerungsraten der „Other goods“) wesentlich geringer ist als in Inflationsraten des Dienstleistungssektors.

Beim Vergleich der BRD und Frankreich, die in etwa über das gleiche Akkumulationsniveau verfügen, sind die höheren französischen Inflationsraten in den 60er Jahren auch aus den sektoralen Strukturdifferenzen erklärbar. Einerseits ist in 
Frankreich der Anteil unterdurchschnittlich produktiver Industriebetriebe wesentlich größer als in der BRD (54), andererseits verfügt die BRD unter den kapitalistischen Industrieländern über den relativ größten Anteil des Industriesektors und über den relativ geringsten Anteil des Dienstleistungssektors am Sozialprodukt, so daß der Inflationsdruck in der BRD aufgrund sektoraler Inhomogenitäten international unterdurchschnittlich ist (55).

Die ungleichen Inflationsraten zwischen den kapitalistischen Industrieländern sind damit insgesamt aus der ungleichen Kapitalrentabilität und den ungleichen sektoralen Strukturen zu erklären. Dabei besteht die Schwierigkeit darin, daß diese beiden Momente auf den Inflationsabstand ungleich entwickelter Länder eine sich konterkarierende Wirkung ausüben. Während aufgrund des Kapitalrentabilitätsabstandes die Inflationsraten in den höher entwickelten Ländern stärker ansteigen als in den weniger entwickelten Ländern, wächst die Inflation aufgrund des höheren Inhomogenitätsgrades der sektoralen Strukturen in den schwächer entwickelten Ländern schneller als in den Ländern. mit höherem Entwicklungsniveau. Für die ungleichmäßige Entwicklung der Inflation zwischen den kapitalistischen Industrieländern im Verlaufe der 60er Jahre kann aufgrund der empirischen Daten generell gesagt werden, daß der sektorale Strukturfaktor das internationale Inflationsgefüge stärker beeinflußt hat als der Kapitalrentabilitätsfaktor. Mit der Verschärfung der Profitabilitätskrise seit Beginn der 70er Jahre hat allerdings der Kapitalrentabilitätsfaktor für die Analyse der ungleichen internationalen Inflationsraten eine größere Bedeutung erlangt. So haben sich Japan und Frankreich von der Spitze des internationalen Inflationsprozesses abkoppeln können und Plätze im Mittelfeld eingenommen, weil ihre Kapitalrentabilität nicht in dem Maße abgenommen hat, wie etwa in Italien und Großbritannien. Für die in den 70er Jahren weiterhin günstige Position der BRD und der USA im Vergleich der internationalen Inflationsraten hat neben dem Kapitalrentabilitätsfaktor (im Bezug auf Großbritannien und Italien) der sektorale Strukturfaktor (in Bezug auf Frankreich und Japan) den Ausschlag gegeben (56).

54 Carre, J.-J./Dubois, P./Malinvaud, E., Abrege de la croissance Francaise, Paris 1973,S.112

55 Zur Bedeutung der sektoralen Strukturdifferenzen für die Inflationsraten Japans vgl. Tasuku Noguchi: Der besondere Charakter der japanischen Inflation, in: Handbuch 4, a.a.O., S. $55 \mathrm{ff}$.

56 Für die Analyse der ungleichmäßigen Entwicklung der Inflation zwischen den kapitalistischen Industrieländern ist zusätzlich zu berücksichtigen, daß die Preissteigerungsraten der Exportwaren in einzelnen Ländern teilweise erheblich von der durchschnittlichen Inflation abweichen. So sind z.B. in den 60er Jahren die Exportpreise Japans im internationalen Vergleich unterdurchschnittlich angestiegen, während die Exportpreise der USA überdurchschnittlich zugenommen haben. Für diese Verschiebungen zwischen ,internen“ und „externen" Inflationsraten ist neben der Währungsposition des jeweiligen Landes (über- oder unterbewertete Währungen) die unterschiedliche Struktur des inländischen und des exportierten ,Warenkorbes" verantwortlich. 


\section{Das Scheitern der Wirtschafts- und Währungsunion und die Perspektiven der europäischen Gemeinschaft}

Nach der Analyse der Ursachen der ungleichmäßigen Entwicklung von Wirtschaftswachstum und Inflation in der Europäischen Gemeinschaft können wir uns jetzt der Beantwortung der am Ende von Abschnitt 1 aufgeworfenen Fragestellungen zuwenden. War das Scheitern der ersten WWU-Etappen der Ausdruck notwendiger Anfangsschwierigkeiten in der Einleitung eines vertieften Integrationsprozesses? Hat die monetäre Integrationskonzeption die erste Phase der Wirtschafts- und Währungsunion überfordert? Kann die WWU durch eine Harmonisierung der Wirtschaftspolitik der Gemieinschaftsländer letztlich doch realisiert werden? Diese Probleme, die die erste Grobanalyse des realen Verlaufes der Wirtschafts- und Währungsunion aufgeworfen hat, gilt es im folgenden zu untersuchen.

Zahlreiche bürgerliche Wirtschaftswissenschaftler sind der Auffassung, daß das vorläufige Scheitern der Wirtschafts- und Währungsunion Ausdruck von Anlaufsschwierigkeiten sowie überzogenen monetären Integrationsansprüchen war, die es durch eine Koordinierung der Wirtschaftspolitik der Mitgliedsländer der EG zu überwinden gelte. So meinen Cairncross, Giersch, Lamfalussy, Petrilli und Uri:

„Unserer Auffassung nach ist die Strategie, die die Währungsunion auf dem Wege über verengte Bandbreiten verwirklichen will, zum Scheitern verurteilt. Es handelt sich dabei im Kern um den Versuch, dieselbe Technik der schrittweisen Annäherung an ein Ziel nach vorgegebenem Zeitplan auf die monetäre Integration anzuwenden, die mit Erfolg bei der Verwirklichung der Zollunion erprobt wurde. Es gibt hier jedoch keine echten Parallelen. Wie die Experten einer europäischen Zentralbank in einer Stellungnahme gegenüber dem Werner-Komitee feststellten, spräche überhaupt viel dafür, die Bandbreiten zu einem Zeitpunkt, zu dem die Konvergenz der Wirtscaftspolitik der Mitglied sländer genügende Fortschritte gemacht hat, in einem Zuge abzuschaffen, anstatt sie stufenweise, d.h. in der ersten Phase verringern zu wollen." (57)

Hans-Eckhart Scharrer resümiert seine Analyse der europäischen Währungspolitik:

„Der Versuch, ein Gemeinschaftssystem mit festen Wechselkursen zu installieren und zu verteidigen, sollte auf absehbare Zeit zurückgestellt werden ... Notwendige Bedingung für eine Stabilisierung der innergemeinschaftlichen Währungsrelationen und schließlich auch für die Gewährleistung des freien Güter- und Faktoraustausches ist eine stärkere Angleichung der Wirtschaftsabläufe der Mitgliedsstaaten. Die traditionelle Konsultationsmethode hat sich als wenig wirkungsvoll erwiesen, um diese Harmonisierung herbeizuführen, da der Rat kontroverse Diskussionen über die Wirtschaftspolitik individueller Länder praktisch stets vermieden hat. Zu prüfen wäre, ob sich nicht durch eine gewisse Regelfindung der nationalen Globalpolitiken und vor allem durch Vorkehrungen zur Verstetigung dieser Politiken bessere Ergebnisse realisieren lassen." (58)

Nun hat aber unsere Analyse der ungleichmäßigen Kapitalakkumulation in der EG überdeutlich gezeigt, daß nicht die unterschiedliche Anwendung des Instrumentariums der Wirtschaftspolitik, sondern strukturelle Komponenten, die von Staatseingriffen nur marginal beeinflußt werden können, für dië divergierenden Wachstums-

57 Cairncross/Giersch/Lamfallussy/Petrilli, Urì: Wirtschaftspolitik für Europa - Wege nach vorn, München und Zürich 1974, S. 47

58 Scharrer, Hans-Eckart, Europäische Währungspolitik vor dem Hintergrund der internationalen Ölpreisexplosion, in:-Köhler/Scharrer (Hrsg.), Die Krise der EG, Hamburg 1974, S. $96 \mathrm{f}$. 
ten des Sozialprodukts und die ungleichen Inflationsraten verantwortlich sind. Die Grenzen staatlicher Wirtschaftspolitik in der Beeinflussung des Akkumulationsverlaufes können in folgenden Punkten zusammengefaßt werden:

1. Staatliche Wirtschaftspolitik ist nicht in der Lage, die strukturellen Komponenten der Kapitalakkumulation wesentlich zu beeinflussen. Die Durchschnittsprofitrate als entscheidender Motor der Kapitalakkumulation wird vor allem durch das Niveau der organischen Zusammensetzung des Kapitals und die Mehrwertrate bestimmt, beides Faktoren, die durch die Eingriffe des Staates nur marginal bestimmt werden können.

2. Stehen sich - wie in der EG - Nationen mit einem ungleichen Entwicklungsniveau gegenüber (BRD versus Italien), dann ist das schwächer entwickelte Land wegen des niedrigeren Niveaus der organischen Zusammensetzung des Kapitals und der damit verbundenen höheren Durchschnittsprofitrate in der Regel zur rascheren Entfaltung der Produktivkraft durch höhere Akkumulationszuwachsraten in der Lage. Dieses ungleiche Akkumulationstempo kann durch eine Koordinierung der Wirtschaftspolitik der beiden Länder nicht verhindert werden, weil diese das Niveaugefälle der organischen Zusammensetzung des Kapitals nicht zu beseitigen vermag.

3. Auch die Auswirkungen einer unterschiedlichen Zweigstruktur der Ökonomie auf das Akkumulationstempo ist durch staatliche Wirtschaftspolitik kaum auszugleichen. Wenn z.B. Frankreich gegenüber der BRD trotz eines 1960 in etwa gleichen industriellen Produktivkraftniveaus im Verlaufe der 60er Jahre rascher und zu günstigeren Bedingungen akkumulieren konnte als die $\mathrm{BRD}$, weil es über eine relativ größere Akkumulationsreserve in Gestalt eines höheren Anteils des Primärsektors am Sozialprodukt verfügte, dann sind die Auswirkungen dieser strukturellen Komponenten auf den Akkumulationsprozeß durch staatliche Interaktionen nicht auszugleichen. Weder kann durch staatliche Politik der unterschiedliche Druck der Reservearmee auf die objektive Kampfkraft der Gewerkschaften wesentlich tangiert werden, also die marginale Gewinnquote verändert werden, noch kann durch eine Koordinierung der Wirtschaftspolitik der in beiden Ländern unterschiedliche Zwang des Kapitals zur Substitution der Arbeitskraft durch Maschinerie, der divergierende Zwang zur Steigerung der organischen Zusammensetzung des Kapitals, ausgeglichen werden.

4. Ebenso sind historisch gewachsene Bedingungen für die Kapitalakkumulation in den einzelnen Ländern, etwa die relative Stagnation des Mezzogiorno in Italien oder der Verlust der Metropolenposition auf dem Weltmarkt wie im Beispiel Großbritanniens, durch staatliche Wirtschaftspolitik im Kern nicht veränderbar. Regionale Wirtschaftsförderungsmaßnahmen und staatliche Exportförderungsmaßnahmen können dem Einfluß derartiger Strukturkomponenten auf den Akkumulationsprozeß allenfalls entgegenwirken, ihn aber nicht grundsätzlich abbauen.

5. Die Folgen eines unterschiedlichen ökonomischen und politischen Bewußtseins der Arbeiterklasse in den EG-Ländern für die Profitquote und damit für das Akkumulationstempo sind ebenso wenig durch eine Koordinierung der staatlichen Wirtschaftspolitik zu beseitigen.

Eine Einkommenspolitik auf EG-Ebene gleicht das unterschiedliche Kampf- 
potential der Gewerkschaften Italiens und Großbritanniens einerseits und der BRD andererseits nicht aus. Das Ausmaß, in welchem sich die Gewerkschaften oder die Gewerkschaftsbasis der einzelnen Länder in das Prokrustesbett staatlicher Lohnregulierungen pressen lassen, wird auch bei einer koordinierten EG-Wirtschaftspolitik weiterhin stark divergieren. Der Kampf zwischen Lohnarbeit und Kapital in seiner unmittelbarsten Form, der Lohnkampf, ist staatlicher Planung nicht zugänglich und schon gar nicht zwischen Ländern mit verschiedenen Traditionen in der Arbeiterbewegung auf ein einheitliches Maß festzulegen.

6. Schließlich hat auch unsere Inflationsanalyse deutlich gemacht, daß nicht die Geld- und Kreditpolitik für das Ausmaß der Steigerungsraten des allgemeinen Preisniveaus verantwortlich gemacht werden kann, vielmehr der Fall der Kapitalproduktivität sowie die sektoralen Strukturdifferenzen den Grad der Inflation bestimmen. Durch den Aufbau einer europäischen Zentralbank und die Delegation der Kompetenzen für die Geld- und Kreditpolitik auf dieses supranationale Organ könnten die Inflationsraten in den verschiedenen EG-Ländern deshalb keineswegs zum Ausgleich gebracht werden.

Den zitierten Wirtschaftswissenschaftlern ist zwar zuzustimmen, wenn sie die Verringerung der Bandbreiten der Wechselkurse bei einer ungleichen ökonomischen Entwicklung als verfrüht kritisieren, sie selbst unterliegen aber der Illusion, daß der Akkumulationsverlauf durch den Staat gesteuert werden könne und von daher durch eine Harmonisierung der nationalen Wirtschaftspolitik der Mitgliedsländer der EG eine gleichgerichtete ökonomische Entwicklung erreichbar sei.

Am Unvermögen der staatlichen Wirtschaftseingriffe gegenüber den strukturellen Komponenten der Kapitalakkumulation müssen sich alle Konzeptionen - ob monetaristische oder ökonomistische - zur Verwirklichung einer Wirtschafts- und Währungsunion in der EG brechen. Angesichts der ungleichen ökonomischen Potentiale der Mitgliedsländer sind alle Versuche, den ökonomischen Integrationsprozeß über die Zollunion hinaus zu vertiefen, zum Scheitern verurteilt.

Die Zollunion konnte in der EG trotz der ungleichen nationalen Entwicklungsniveaus durchgesetzt werden, weil sie prinzipiell bei einer Verschiebung der Konkurrenzposition der Mitgliedsländer untereinander die Möglichkeit zu Wechselkurskorrekturen offenläßt. In der Wirtschafts- und Währungsunion wird den einzelnen $\mathrm{Na}$ tionen dieser Schutz in Gestalt der Wechselkurse genommen, die WWU setzt deshalb eine gleiche ökonomische Entwicklung voraus, soll sie nicht auf Kosten der sich schwächer entwickelnden nationalen Kapitale realisiert werden. Da eine gleichmäßige Kapitalakkumulation, wie wir gesehen haben, in der EG nicht herstellbar ist, kann eine Wirtschafts- und Währungsunion nur noch durch außerökonomischen Zwang, durch den politisch-militärischen Druck einer imperialistischen Führungsmacht durchgesetzt werden. Beim momentanen und absehbaren Kräfteverhältnis in der EG verfügt aber kein Mitgliedsland, auch nicht die BRD, über das Machtpotential, das für eine zwangsweise Integration Europas notwendig wäre.

Der westeuropäische Integrationsprozeß ist damit seit der Realisierung der Zollunion und des Gemeinsamen Agrarmarktes Ende der 60er Jahre in eine Stagnationsphase eingetreten, die trotz verschiedener Anläufe der EG-Länder auf den Gip- 
felkonferenzen in Den Haag (Dezember 1969), in Paris (Oktober 1972 und Dezember 1974) sowie in Rom (Frühjahr 1977) nicht überwunden werden konnte. Diese Krise der EG ist mit den bisher üblichen Schwierigkeiten des Integrationsprozesses, die in zahlreichen Sektoren der Integrationspolitik, der Agrarpolitik, der Forschungspolitik, der Energiepolitik usw. permanent auftraten, und in der Öffentlichkeit das Bewußtsein einer europäischen Dauerkrise erzeugten, keineswegs vergleichbar. Die Krise der Wirtschafts- und Währungsunion hat vielmehr angesichts ungleicher ökonomischer Potentiale die prinzipiellen Schranken eines zwischenstaatlichen Integrationsprozesses mit friedlichen Mitteln offenbart. Für den bisher erreichten Integrationsstand kann das Scheitern der Wirtschafts- und Währungsunion ebenfalls tiefgreifende Folgen haben. Die auf halbem Wege stecken gebliebene Integration der EG birgt potentiell stets die Gefahr des Rückfalls in die totale Nationalstaatlichkeit in sich. Insbesondere in einer Periode tiefer Akkumulationskrisen in allen Mitgliedsstaaten, wie wir sie seit 1974 erleben, droht der Rückfall hinter den Gemeinsamen Markt, in den Protektionismus, permanent. Nach dem italienischen Import-Bardepot von 1974 bis 1976, den dänischen Verbrauchssteuererhöhungen für eine Reihe von Importwaren im Jahre 1974 und den französisch-italienischen Weinkriegen der Jahre 1975/76, Maßnahmen, die die Grundpfeiler der bisherigen EG-Integration antasteten, wurde im Sommer 1977 das Protektionismusproblem in der EG durch Forderungen der Textil-, Papier- und Stahlindustrie nach Importrestriktionen erneut virulent. Insbesondere in Frankreich drängen diese Kapitalfraktionen auf staatliche Schutzmaßnnahmen, die, wenn sie von der EG insgesamt nicht übernommen werden, von Frankreich einseitig verfügt werden sollen. Da von der konjunkturellen Belebung nach der Weltwirtschaftskrise 1974/76 zahlreiche Industriebranchen nur zögernd erfaßt wurden und das Arbeitslosenproblem in der kapitalistischen Welt nirgendwo gelöst werden konnte, können verstärkte protektionistische Tendenzen in einzelnen kapitalistischen Industrieländern jederzeit auf die Tagesordnung treten und den vielbeschworenen „Rückfall in die 30er Jahre "Wirklichkeit werden lassen. 